\title{
Hermitian-Einstein metrics and Chern number inequalities on parabolic stable bundles over Kähler manifolds
}

\author{
LI JIAYU
}

\begin{abstract}
Let $\bar{X}$ be a compact complex manifold with a smooth Kähler metric and $D=\sum_{i=1}^{m} D_{i}$ a divisor in $\bar{X}$ with normal crossings. Let $E$ be a holomorphic vector bundle over $\bar{X}$ with a stable parabolic structure along $D$. We prove that there exists a Hermitian-Einstein metric on $E^{\prime}=\left.E\right|_{\bar{X} \backslash D}$ and obtain a Chern number inequality for a stable parabolic bundle.

Without the assumption that the irreducible components $D_{i}$ of $D$ meet transversely, using Hironaka's theorem on the resolution of singularities, we also get a Chern number inequality for a more general stable parabolic bundle.
\end{abstract}

\section{Introduction.}

Let $\bar{X}$ be a compact Kähler manifold of complex dimension $n$ with a Kähler form $\omega$. Let $E$ be a rank $r$ holomorphic vector bundle over $\bar{X}$. It is proved that $E$ is stable if and only if $E$ admits a Hermitian-Einstein metric, under the assumption that $E$ is indecomposable ([N-S], [D1], [D2], [D3], [U-Y]). The theorem yields Bogomolov-Gieseker inequality easily, which says that, if $E$ is stable,

$$
\left(2 C_{2}(E)-\frac{r-1}{r} C_{1}(E)^{2}\right) \cdot[\omega]^{n-2} \geq 0 .
$$

Let $D=\sum_{i=1}^{m} D_{i}$ be a divisor in $\bar{X}$ with normal crossings, we introduce a parabolic structure of $E$ with respect to $D$ which consists of flags of $\left.E\right|_{D_{i}}$ and weights attached to the flags, we define the notion of parabolic stability of a parabolic structure (see Section 2). Set $X=\bar{X} \backslash D, E^{\prime}=\left.E\right|_{X}$. In section 3 , we construct a metric $K_{0}$ on $E^{\prime}$ with the property that $\left|F_{K_{0}}\right|_{K_{0}} \in L^{p}(X)$ $(p>1)$. In Section 4 , we construct Kähler metrics $\omega_{\alpha}(0<\alpha<2)$ on $X$ and show that $\left(X, \omega_{\alpha}\right)$ satisfies the three assumptions in Section 2 of [S1]. It is proved in Section 5 (Proposition 5.10) that the parabolic structure is parabolic stable if and only if $\left(E, K_{0}\right)$ is analytic stable (Definition 5.2, also 
see [S1],p.877), which yields one of our main results (Theorem 6.3) that the parabolic stability of a parabolic structure is essentially equivalent to the existence of a Hermitian-Einstein metric on $E^{\prime}$ with respect to $\omega_{\alpha}$ for some $0<\alpha<2$. Furthermore, we prove a Chern number inequality in section 7 (Theorem 7.5) for a stable parabolic structure.

In section 8 , we prove a Chern number inequality (Theorem 8.3 ) for a more general stable parabolic structure. In this case, we do not assume that the irreducible components $D_{i}$ of $D$ meet transversely, and we need not suppose that the flags of $\left.E\right|_{D_{i}}$ satisfy the compatibility condition (Definition 2.1 and Definition 8.1). We use a theorem of Hironaka $[H]$ on the resolution of singularities to get complex manifold $\widetilde{X}$ by successively blowing up submanifolds such that the proper transforms $D_{i}^{*}$ of $D_{i},(i=1, \cdots, m)$ do not meet each other. Let $q: \widetilde{X} \rightarrow \bar{X}$ be the canonical map, and let $\widetilde{\omega}$ be a Kähler form on $\widetilde{X}$. We show (Lemma 8.5) that the stable parabolic structure of $E$ along $D=\sum_{i=1}^{m} D_{i}$ induces a stable parabolic structure of $q^{*} E$ along $D^{*}=\sum_{i=1}^{m} D_{i}^{*}$ on $\widetilde{X}$ using the Kähler form $q^{*} \omega+\varepsilon \widetilde{\omega}$ for sufficiently small $\varepsilon>0$. Then Theorem 7.5 yields another main result of this paper, the Chern number inequality Theorem 8.3.

If $D$ is a smooth divisor, Li-Narasimhan [L-N] construct a metric $K_{0}$ on. $E^{\prime}$ with $\left|F_{K_{0}}\right|_{K_{0}} \in L^{p}(X)(p>2)$. If $X$ is of complex dimension 2, they show the equivalence between the stability of a parabolic structure and the existence of a Hermitian-Einstein metric on the bundle, using the restriction of the Kähler metric $\omega$ to $X$.

Parabolic bundles over Riemann surfaces is treated in [MS, B, K, Na-St, P, S1, S2].

Acknowledgement. The author would like to express his gratitude to Prof. G. Tian for his advising in the construction of the metrics on the bundle. He would like to express his gratitude to Prof. M. S. Narasimhan for his explaining the Hironaka's theorem.

\section{Parabolic stability over Kähler manifolds.}

Let $\bar{X}$ be a compact Kähler manifold of complex dimension $\mathrm{n}$ with a Kähler metric $\omega, D$ a divisor in $\bar{X}$ with normal crossings. Let $X=\bar{X} \backslash D$, the restriction of $\omega$ to $X$ gives a Kähler metric on $X$, we fix it once for all. Set $D=\sum_{i=1}^{m} D_{i}$ where the irreducible components $D_{i}$ of $D$ are smooth and meet transversely.

Let $E$ be a holomorphic vector bundle over $\bar{X}, E^{\prime}=\left.E\right|_{X}$. Define $I$ to be 
the set of all tuples of integers $\left(k_{1}, \cdots, k_{j}\right)$ with $1 \leq j \leq n$ and $1 \leq k_{i} \leq m$. For each $J=\left(k_{1}, \cdots, k_{j}\right) \in I$, denote by $X_{J}$ the smooth variety defined as the intersection of $D_{k_{1}} \cdots D_{k_{j}}$.

Definition 2.1. A parabolic structure on $\mathrm{E}$ with respect to $D$ consists of

a) flags of $\left.E\right|_{D_{i}}(1 \leq i \leq m)$ :

$$
\left.E\right|_{D_{i}}=F_{1}^{i} \supset F_{2}^{i} \supset \cdots \supset F_{m_{i}}^{i} \supset\{0\}=F_{m_{i}+1}^{i}
$$

where $F_{l+1}^{i}$ is a proper subbundle of $F_{l}^{i}\left(1 \leq l \leq m_{i}-1\right)$, and the flags satisfy the following compatibility condition: For every $J=\left(k_{1}, \cdots, k_{j}\right) \in I,\left\{\left.F_{l}^{k_{i}}\right|_{D_{k_{1}} \cdots D_{k_{j}}}, 1 \leq i \leq j, 1 \leq l \leq m_{k_{i}}\right\}$ yields a flag of $\left.E\right|_{D_{k_{1}} \cdots D_{k_{j}}}$ which is a refined flag of $\left\{\left.F_{l}^{k_{i}}\right|_{D_{k_{1}} \cdots D_{k_{j}}}, 1 \leq l \leq m_{k_{i}}\right\}$ for each $i \in\{1, \cdots, j\}$.

b) weights $\alpha_{1}^{i}, \alpha_{2}^{i}, \cdots, \alpha_{m_{i}}^{i}$ attached to $F_{1}^{i}, F_{2}^{i}, \cdots, F_{m_{i}}^{i}$, satisfying $0 \leq$ $\alpha_{1}^{i}<\alpha_{2}^{i}<\cdots<\alpha_{m_{i}}^{i}<1$.

A holomorphic vector bundle $E$ with a parabolic structure is called parabolic bundle.

Definition 2.2. We define the parabolic degree of a parabolic bundle $E$ by

$$
p a r \operatorname{deg} E=\operatorname{deg} E+\sum_{i=1}^{m} \sum_{l=1}^{m_{i}} \operatorname{rank}\left(F_{l}^{i} / F_{l+1}^{i}\right) \alpha_{l}^{i} \operatorname{deg}\left[D_{i}\right]
$$

where $\left[D_{i}\right]$ is the line bundle defined by the divisor $D_{i}, \operatorname{deg} E$ (resp. $\operatorname{deg}\left[D_{i}\right]$ ) is the degree of $E$ (resp. the degree of $\left[D_{i}\right]$ ) in the usual sense using the Kähler form $\omega$.

Suppose that $V$ is a proper coherent subsheaf of $E$ with quotient torsion free. Then there is a natural flag of $\left.V\right|_{D_{i}}$ by coherent subsheaves

$$
\left.V\right|_{D_{i}}=F_{1}^{i} V \supset \cdots \supset F_{n_{i}}^{i} V \supset\{0\}=F_{n_{i}+1}^{i} V
$$

induced by $F_{1}^{i} \cap V \supseteq \cdots \supseteq F_{m_{i}}^{i} \cap V \supseteq\{0\}$, clearly, $n_{i} \leq m_{i}$. Let us define the weights attached to the flag by $\alpha_{l}^{i}(V)=$ the largest $\alpha_{k}^{i}$ such that $F_{l}^{i} V \subseteq F_{k}^{i} \cap V$, i.e., the subscript $k$ is the largest integer with the property that $F_{l}^{i} V \subseteq F_{k}^{i}, 1 \leq l \leq n_{i}, 1 \leq i \leq m$. 
Definition 2.3. We define the parabolic degree of $V$ by

$$
p a r \operatorname{deg} V=\operatorname{deg} V+\sum_{i=1}^{m} \sum_{l=1}^{n_{i}} \operatorname{rank}\left(F_{l}^{i} V / F_{l+1}^{i} V\right) \alpha_{l}^{i}(V) \operatorname{deg}\left[D_{i}\right]
$$

Definition 2.4. We say that a parabolic bundle $E$ is parabolic stable if for every proper coherent subsheaf $V$ of $E$ with quotient torsion free we have

$$
\frac{\operatorname{par} \operatorname{deg} V}{\operatorname{rank} V}<\frac{\operatorname{par} \operatorname{deg} E}{\operatorname{rank} E}
$$

\section{Construction of metrics on vector bundles.}

Let $E$ be a parabolic bundle over $\bar{X}$ as given in Section 2. At a point $p \in D$ through which $j(1 \leq j \leq n)$ of the $D_{i}$ pass, we may choose local holomorphic coordinates in a neighborhood $U=\Delta^{n}=\left\{\left|z_{i}\right|<1, i=1, \cdots, n\right\}$ of $p=$ $(0, \cdots, 0)$ such that $D \cap U=\left\{z_{1} \cdots z_{j}=0\right\}$ is the union of coordinate hyperplanes. The complement $U^{*}=U \backslash U \cap D=\left(\triangle^{*}\right)^{j} \times \triangle^{n-j}$ is a punctured polycylinder $P^{*}(j, n)$ given by $\left\{\left(z_{1}, \cdots, z_{n}\right)|| z_{i} \mid<1, z_{1} \cdots z_{j} \neq 0\right\}$.

Definition 3.1. If $\left\{e_{1}, \cdots, e_{r}\right\}(r=\operatorname{rank} E)$ is a holomorphic basis of $E$ in $\triangle^{n}$ satisfying the property that, for any $i \in\{1, \cdots, j\}$ :

$$
\begin{aligned}
& \left\{e_{r-r_{m_{i}}^{i}+1}, \cdots, e_{r}\right\} \text { is a basis of } F_{m_{i}}^{i} \text { over } U \cap D_{i}, \\
& \left\{e_{r-r_{m_{i}-1}^{i}+1}, \cdots, e_{r}\right\} \text { is a basis of } F_{m_{i}-1}^{i} \text { over } U \cap D_{i}, \\
& \vdots \\
& \left\{e_{r-r_{2}^{i}+1}, \cdots, e_{r}\right\} \text { is a basis of } F_{2}^{i} \text { over } U \cap D_{i}
\end{aligned}
$$

where $r_{l}^{i}=\operatorname{rankF}_{l}^{i}, l=1, \cdots, m_{i}$, we say that it preserves the flags on $U \cap D$.

The following lemma is clear.

Lemma 3.2. There is a holomorphic basis $\left\{e_{1}, \cdots, e_{r}\right\}$ of $E$ in $\Delta^{n}$ such that it preserves the flags on $U \cap D$.

Proof. We choose a holomorphic basis $\left\{e_{1}, \cdots, e_{r}\right\}$ of $\left.E\right|_{D_{1} \cdots D_{j}}$, such that it preserves the flag $F$ yielded by $\left\{\left.F_{l}^{i}\right|_{D_{1} \ldots D_{j}}, 1 \leq l \leq m_{i}, 1 \leq i \leq j\right\}$. Since 
$F$ is a refined flag of $\left\{\left.F_{l}^{i}\right|_{D_{1} \cdots D_{j}}, 1 \leq l \leq m_{i}\right\}$ for each $i \in\{1, \cdots, j\}$, the basis $\left\{e_{1}, \cdots, e_{r}\right\}$ can be extended naturally so that it preserves the flags on $U \cap D$. This proves the lemma.

Let $\left\{e_{1}, \cdots, e_{r}\right\}$ be a holomorphic basis of $E$ in a neighborhood $U_{p_{1}}$ of $p_{1},\left\{f_{1}, \cdots, f_{r}\right\}$ a holomorphic basis of $E$ in a neighborhood $U_{p_{2}}$ of $p_{2}$. Suppose that $\left(f_{1}, \cdots, f_{r}\right)=\left(e_{1}, \cdots, e_{r}\right) g$, i.e. $f_{\beta}=e_{\alpha} g_{\alpha \beta}$ in $U_{p_{1}} \cap U_{p_{2}} \neq \emptyset$, $U_{p_{1}} \cap U_{p_{2}} \cap D=\cup_{i=1}^{j} D_{k_{i}}(1 \leq j \leq n)$. Assume that $\left\{e_{1}, \cdots, e_{r}\right\}$ (resp. $\left.\left\{f_{1}, \cdots, f_{r}\right\}\right)$ preserves the flags in $U_{p_{1}}$ (resp. in $\left.U_{p_{2}}\right)$. Then on each $D_{k_{i}}(i=$ $1,2, \cdots, j)$

$$
g_{\alpha \beta}=0 \text { if } r-r_{l-1}^{k_{i}}+1 \leq \beta \leq r-r_{l}^{k_{i}}, \quad \alpha \leq r-r_{l-1}^{k_{i}}
$$

here $r_{l}^{k_{i}}=\operatorname{rank} F_{l}^{k_{i}}, 2 \leq l \leq m_{k_{i}}+1$.

For each $i=1, \cdots, m$, we choose a metric on the line bundle $\left[D_{i}\right]$ defined by the divisor $D_{i}$. Let $\sigma_{i}$ be the canonical section of $D_{i}$ which vanishes on $D_{i}$. We may assume that its langth $\left|\sigma_{i}\right|<1$. We put $\sigma=\sigma_{i} \otimes \cdots \otimes \sigma_{m}$, which is a section of $[D]$, then $|\sigma|=\Pi_{i}\left|\sigma_{i}\right|<1$. Set

Put $\beta_{l}^{i}=\alpha_{j}^{i}$ if $r-r_{j}^{i}<l \leq r-r_{j+1}^{i}$, where $r_{j}^{i}=\operatorname{rank} F_{j}^{i}, j=1, \cdots, m_{i}$.

$$
\begin{aligned}
\beta^{i} & =\left(\begin{array}{ccc}
\sigma_{i}^{-\beta_{1}^{i}} & & \\
& \ddots & \\
& & \sigma_{i}^{-\beta_{r}^{i}}
\end{array}\right) \\
S^{i} & =\left(\begin{array}{ccc}
\left|\sigma_{i}\right|^{\beta_{1}^{i}} & & \\
& \ddots & \\
& & \left|\sigma_{i}\right|^{\beta_{r}^{i}}
\end{array}\right)
\end{aligned}
$$

Now we construct a metric $H$ on $\left.E\right|_{P^{*}(j, n)}$. Let $\left\{e_{1}, \cdots, e_{r}\right\}$ be a holomorphic basis of $E$ in $U$ preserving the flags on $U \cap D$. Assume that $U \cap D=\cup_{i=1}^{j} D_{k_{i}}$, we define the metric $H$ so that its matrix with respect to $\left\{e_{1}, \cdots, e_{r}\right\}$ is $\left(S^{k_{1}}\right)^{2} \cdots\left(S^{k_{j}}\right)^{2}$. Set $\left(e_{1}^{k_{1} \cdots k_{j}}, \cdots, e_{r}^{k_{1} \cdots k_{j}}\right)=\left(e_{1}, \cdots, e_{r}\right) \beta^{k_{1}} \cdots \beta^{k_{j}}$, it is well defined in a small neighborhood of any point $x \in P^{*}(j, n)$, and it is a holomorphic basis of $E^{\prime}$ there. It is clear that with respect to the basis $\left(e_{1}^{k_{1} \cdots k_{j}}, \cdots, e_{r}^{k_{1} \cdots k_{j}}\right)$ the matrix of $H$ is identity.

There are finite neighborhoods $U_{i}$ and $V_{i}(i=1, \cdots, N)$ such that, 1$)$ $U_{i} \supset \supset V_{i}$ and $\left.\cup_{i} V_{i} \supset D ; 2\right)$ associated to each $U_{i}$ there is a unique $j$-tuple $\left(k_{1}, \cdots, k_{j}\right)$ with $1 \leq j \leq n$ such that $U_{i} \cap D_{k_{1}} \cap \cdots \cap D_{k_{j}}$ is an open coordinate chart of $D_{k_{1}} \cap \cdots \cap D_{k_{j}}$ disjoint from any other $D_{k}\left(k \neq k_{l}\right.$, $l=1, \cdots, j)$. Let $U_{0}=\bar{X} \backslash \cup_{i} V_{i}$. Then $\left\{U_{i} \mid i=0, \cdots, N\right\}$ is a finite 
covering of $\bar{X}$. Suppose that $\left\{\psi_{i} \in C_{0}^{\infty}\left(U_{i}\right) \mid i=0, \cdots, N\right\}$ is a partition of unity corresponding to the covering. In each $U_{i}(i=1, \cdots, N)$ we choose a. metric $H_{i}$ on $E^{\prime}$ as above and in $U_{0}$ we choose a smooth metric on $E$. We define a metric $K_{0}$ on $E^{\prime}$ by

$$
K_{0}=\sum_{i=0}^{N} \psi_{i} H_{i}
$$

Lemma 3.3. Let $K_{0}$ be the metric on $E^{\prime}$ defined above, then its curvature form satisfied that $\left|F_{K_{0}}\right|_{K_{0}} \in L^{p}(X)(p>1)$.

Proof. It is clear that, in $U_{k} \backslash\left(\cup_{i \neq k} U_{i}\right)(k=1, \cdots, N),\left|F_{K_{0}}\right|_{K_{0}} \in L^{\infty}$. Suppose that $U_{i_{1}} \cap U_{i_{2}} \supset D_{k_{1}}\left(1 \leq i_{1} \leq N, 1 \leq i_{2} \leq N\right), D_{k_{1}} \cdots D_{k_{j_{1}}}$ is assoiated to $U_{i_{1}}, D_{k_{1}} D_{l_{2}} \cdots D_{l_{j_{2}}}$ is associated to $U_{i_{2}}$. Let $\left\{e_{1}, \cdots, e_{r}\right\}$ be a holomorphic basis of $E$ preserving the flags on $U_{i_{1}} \cap D$, and let $\left\{f_{1}, \cdots, f_{r}\right\}$ be a holomorphic basis of $E$ preserving the flags on $U_{i_{2}} \cap D$. We write $f_{\beta}=e_{\alpha} g_{\alpha \beta}$, then

$$
\begin{aligned}
\left(f_{1}^{k_{1} l_{2} \cdots l_{j_{2}}}, \cdots, f_{r}^{k_{1} l_{2} \cdots l_{j_{2}}}\right) & \\
= & \left(f_{1} \cdots f_{r}\right) \beta^{k_{1}} \beta^{l_{2}} \cdots \beta^{l_{j_{2}}}=\left(e_{1} \cdots e_{r}\right) g \beta^{k_{1}} \beta^{l_{2}} \cdots \beta^{l_{j_{2}}} \\
& =\left(e_{1}^{k_{1} \cdots k_{j_{1}}}, \cdots, e_{r}^{k_{1} \cdots k_{j_{1}}}\right)\left(\beta^{k_{j_{1}}}\right)^{-1} \cdots\left(\beta^{k_{2}}\right)^{-1}\left(\beta^{k_{1}}\right)^{-1} g \beta^{k_{1}} \beta^{l_{2}} \cdots \beta^{l_{j_{2}}}
\end{aligned}
$$

where $g$ is the matrix with elements $g_{\alpha \beta}$.

Set

$$
G=\left(\beta^{k_{1}}\right)^{-1} g \beta^{k_{1}}=\left(G_{\alpha \beta}\right)
$$

By (1) we can see that $G_{\alpha \alpha}$ are able to be extended holomorphically to $D_{k_{1}}$ and that $G_{\alpha \beta}=H_{\alpha \beta} \sigma_{k_{1}}^{\gamma_{\alpha \beta}}(\alpha \neq \beta)$ where

$$
\gamma_{\alpha \beta} \geq \min \left\{\alpha_{i}^{k_{1}}-\alpha_{i-1}^{k_{1}}, 1-\left(\alpha_{i}^{k_{1}}-\alpha_{i-1}^{k_{1}}\right) \mid i=2, \cdots, m_{k_{1}}+1\right\}>0
$$

and $H_{\alpha \beta}$ are able to be extended holomorphically to $D_{K_{1}}$. So, using the holomorphic basis $\left(e_{1}^{k_{1} \cdots k_{j_{1}}}, \cdots, e_{r}^{k_{1} \cdots k_{j_{1}}}\right)$, applying the fact that $G_{\alpha \beta}$ are holomorphic away from the divisor, we can calculate the curvature form and obtain

$$
F_{K_{0}}=a_{k_{1} \bar{k}_{1}}\left|\sigma_{k_{1}}\right|^{2(\gamma-1)} d z^{k_{1}} d \bar{z}^{k_{1}}+A
$$

where

$$
\gamma \geq \min \left\{\alpha_{i}^{k_{1}}-\alpha_{i-1}^{k_{1}}, 1-\left(\alpha_{i}^{k_{1}}-\alpha_{i-1}^{k_{1}}\right) \mid i=2, \cdots, m_{k_{1}}+1\right\}>0,
$$


$A$ is smooth, $a_{k_{1} \overline{k_{1}}}$ is smooth.

Similarly, if $\cap_{k=1}^{k_{0}} U_{i_{k}} \supset \cup_{j=1}^{l} D_{k_{j}}$, we can show that, in $\cap_{k=1}^{k_{0}} U_{i_{k}}$,

$$
F_{K_{0}}=\sum_{j=1}^{l} a_{k_{j} \bar{k}_{j}}\left|\sigma_{k_{j}}\right|^{2\left(\gamma_{j}-1\right)} d z^{k_{j}} d \bar{z}^{k_{j}}+A
$$

where

$$
\gamma_{j} \geq \min \left\{\alpha_{i}^{k_{j}}-\alpha_{i-1}^{k_{j}}, 1-\left(\alpha_{i}^{k_{j}}-\alpha_{i-1}^{k_{j}}\right) \mid i=2, \cdots, m_{k_{j}}+1\right\}>0,
$$

$a_{k_{j} \bar{k}_{j}}$ is smooth $(j=1, \cdots, l), A$ is smooth. Therefore the lemma follows.

\section{Singular metrics on manifolds.}

Recall that $\omega$ is a Kähler metric of $\bar{X}$. For $0<\alpha<2$ we define

$$
\omega_{\alpha}=\sqrt{-1}\left(\frac{2}{2-\alpha}\right) \sum_{i=1}^{m} \partial \bar{\partial}\left|\sigma_{i}\right|^{2-\alpha}+C_{\alpha} \omega
$$

where $C_{\alpha}$ is a constant large enough so that $\omega_{\alpha}$ is a Kähler metric on $X$. We set $\omega_{0}=\omega$.

For any point $p \in D$, we choose a neighborhood $U_{p}$ of $p$. Assume that $\left(z_{1}, \cdots, z_{n}\right)$ is a coordinate system in $U_{p}$ such that $U_{p} \cap D=\left\{z_{1} \cdots z_{j}=0\right\}$ Then we can see that, in $U_{p} \backslash D, \omega_{\alpha}$ is quasi isomeric to

$$
\sum_{i=1}^{j}\left|\sigma_{i}\right|^{-\alpha} d z_{i} \wedge d \bar{z}_{i}+\sum_{i=j+1}^{n} d z_{i} \wedge d \bar{z}_{i}
$$

Applying the weighted Sobolev inequality proved in [St] (Theorem 2.2.56), we obtain, for any $f \in C^{\infty}(\bar{X})$

$$
\left(\int_{M}|f|^{r}|\sigma|^{-\alpha} d V\right)^{\frac{1}{r}} \leq C_{\alpha}\left(\left(\int_{M}|\nabla f|^{2} d V\right)^{\frac{1}{2}}+\left(\int_{M}|f|^{2}|\sigma|^{-\alpha} d V\right)^{\frac{1}{2}}\right)
$$

if $2 \leq r \leq \frac{2 n-\alpha}{n-1}$, where $d V$ is the volume element of $\omega, \nabla$ is the gradient with respect to $\omega$.

Since in $U_{p} \backslash D,|\nabla f|^{2}=\sum_{i=1}^{n}\left|\frac{\partial f}{\partial z_{i}}\right|^{2}$,

$$
\begin{aligned}
|\nabla \alpha f|^{2} & \geq C_{\alpha}\left(\sum_{i=1}^{j}\left|\sigma_{i}\right|^{\alpha}\left|\frac{\partial f}{\partial z_{i}}\right|^{2}+\sum_{i=j+1}^{n}\left|\frac{\partial f}{\partial z_{i}}\right|^{2}\right) \\
& \geq C_{\alpha}|\sigma|^{\alpha}|\nabla f|^{2}
\end{aligned}
$$


we have the Sobolev inequality:

$$
\left(\int_{M}|f|^{r} d V_{\alpha}\right)^{\frac{1}{r}} \leq C_{\alpha}\left(\left(\int_{M}|\nabla \alpha f|^{2} d V_{\alpha}\right)^{\frac{1}{2}}+\left(\int_{M}|f|^{2} d V_{\alpha}\right)^{\frac{1}{2}}\right)
$$

if $2 \leq r \leq \frac{2 n-\alpha}{n-1}$, where $d V_{\alpha}$ is the volume element of $\omega_{\alpha}$, and $\nabla \alpha$ is the gradient with respect to $\omega_{\alpha}$.

Therefore, we have the following proposition.

Proposition 4.1. $\left(X, \omega_{\alpha}\right)$ satisfies the three assumptions in [S1] (Section 2), that is, (1) $\left(X, \omega_{\alpha}\right)$ has finite volume; (2) there exists an exhaustion function $\phi$ with $\triangle_{\alpha} \phi$ bounded; (3) if $f$ is a nonnegative bounded function on $X$ with $\triangle_{\alpha} f \geq-B\left(B \in L^{p}(X), p>n\right)$, then $\|f\|_{L^{\infty}(X)} \leq C\left(\|B\|_{L^{p}(X)}+\right.$ $\left.\|f\|_{L^{1}(X)}\right)$ where $\triangle_{\alpha}$ is the Laplace operator with respect to $\omega_{\alpha}$.

Proof. Assumption (1) is clearly satisfied. We set $\phi=\log |\sigma|^{2}$. Then $\triangle_{\alpha} \phi=$ $-\sqrt{-1} \Lambda_{\alpha} \bar{\partial} \partial \phi$. The Poincaré-Lelong formula ([SABK], Ch. II, Section 1, Theorem 2) yields that it satisfies Assumption (2). To prove Assumption (3) is satisfied, it suffices to show that the Moser's iterative argument [Mo] (also see [G-T], Ch. 8) works on the manifold $\left(X, \omega_{\alpha}\right)$, which is guaranteed by the Sobolev inequality that we proved above.

\section{Analytic stability and parabolic stability.}

Let $E^{\prime}=\left.E\right|_{X}, K$ a Hermitian metric on $E^{\prime}$, let $d_{K}=\partial_{K}+\bar{\partial}$ be the Hermitian connection of $K, F_{K}$ the curvature of $d_{K}$.

If $\left|\Lambda_{\alpha} F_{K}\right|_{K} \in L^{1}\left(X, \omega_{\alpha}\right),(0 \leq \alpha<2)$, where $\Lambda_{\alpha}$ is the contraction with respect to the Kähler form $\omega_{\alpha}$, we can define (see [L-N], [S1]) the analytic degree of $(E, K)$ by

$$
\begin{aligned}
d_{\alpha}(E, K) & =\frac{\sqrt{-1}}{2 \pi} \int_{X} \operatorname{tr}\left(\Lambda_{\alpha} F_{K}\right) d V_{\alpha} \\
& =\frac{\sqrt{-1}}{2 \pi} \int_{X} \operatorname{tr} F_{K} \wedge * \omega_{\alpha} \\
& =\frac{\sqrt{-1}}{2 \pi} \int_{X} \operatorname{tr} F_{K} \wedge \frac{\omega_{\alpha}^{n-1}}{(n-1) !}
\end{aligned}
$$

If $V$ is a proper coherent subsheaf of $E^{\prime}$ with quotient torsion free, we can 
define the analytic degree of $V$ (see [L-N], [S1]) by

$$
\begin{aligned}
d_{\alpha}(V, K) & =\frac{\sqrt{-1}}{2 \pi} \int_{X} \operatorname{tr}\left(\Lambda_{\alpha} F_{\left.K\right|_{V}}\right) d V_{\alpha} \\
& =\frac{\sqrt{-1}}{2 \pi} \int_{X} \operatorname{tr}\left(\pi \Lambda_{\alpha} F_{K}\right) d V_{\alpha}-\frac{1}{2 \pi} \int_{X}|\bar{\partial} \pi|_{K, \omega_{\alpha}}^{2} d V_{\alpha}
\end{aligned}
$$

where $\pi$ is the orthogonal projection with respect to the metric $K$ onto $V$ in the complement of an analytic set. The analytic set is of codimension $\geq 2$, outside which $V$ is a proper subbundle of $E^{\prime}$.

Let $S_{K}=S_{K}\left(E^{\prime}\right)$ denote the vector bundle of self-adjoint endmorphisms of $E^{\prime}$ with respect to $\mathrm{K}$.

Definition 5.1 ([L-N]). Suppose that $K, H$ are Hermitian metrics on $E^{\prime}$ with the property that $\left|\Lambda_{\alpha} F_{K}\right|_{K} \in L^{1}\left(X, \omega_{\alpha}\right)$ and $\left|\Lambda_{\alpha} F_{H}\right|_{H} \in L^{1}\left(X, \omega_{\alpha}\right)$. Let $H=K h$. If

a) $H, K$ are mutually bounded;

b) $|\bar{\partial} h|_{K, \omega_{\alpha}} \in L^{2}\left(X, \omega_{\alpha}\right)$ then we say that $H$ and $K$ are compatible with respect to $\omega_{\alpha}$.

It is proved in [L-N] (Lemma 3.4) that, if $K$ and $H$ are compatible with respect to $\omega_{\alpha}$, then $d_{\alpha}(E, K)=d_{\alpha}(E, H)$.

Definition 5.2. Suppose that $K$ is a Hermitian metric on $E^{\prime}$ with the property that $\left|\Lambda_{\alpha} F_{K}\right|_{K} \in L^{1}\left(X, \omega_{\alpha}\right)$. We say that $(E, K)$ is analytic stable with respect to $\omega_{\alpha}$, if for every proper coherent subsheaf $V$ of $E^{\prime}$ with quotient torsion free,

$$
\frac{d_{\alpha}(V, K)}{\operatorname{rankV}}<\frac{d_{\alpha}(E, K)}{\operatorname{rankE}}
$$

We compute the analytic degree of $\left(E, K_{0}\right)$ with respect to $\omega_{\alpha}$ where $K_{0}$ is defined in Section 3. For this purpose, we introduce a metric $K_{1}$ of $E$ over $\bar{X}$. We adopt the same notations as that in Section 3 . Let $H^{\prime}$ be a metric on $\left.E\right|_{\Delta^{n}}$ whose matrix with respect to the basis $\left\{e_{1}, \cdots, e_{r}\right\}$ is identity. Then we choose, in each $U_{i}(i=1, \cdots, N)$, a metric $H_{i}^{\prime}$ on $E$ as above, and define the metric $K_{1}$ by

$$
K_{1}=\psi_{0} h_{0}+\sum_{i=1}^{N} \psi_{i} h_{i}^{\prime}
$$

Proposition 5.3. $d_{0}\left(E, K_{0}\right)=\operatorname{par} \operatorname{deg} E$ 
Proof. Set $K_{1}^{-1} K_{0}=h$, then $\operatorname{tr} F_{K_{0}}=\operatorname{tr} F_{K_{1}}+\bar{\partial} \partial \log \operatorname{det} h$.

$$
d_{0}\left(E, K_{0}\right)=\operatorname{deg} E+\frac{\sqrt{-1}}{2 \pi} \int_{X} \bar{\partial} \partial \log \operatorname{det} h \wedge * \omega
$$

We have

$$
\begin{aligned}
d_{0}\left(E, K_{0}\right)= & \operatorname{deg} E+\frac{\sqrt{-1}}{2 \pi} \int_{X} \bar{\partial} \partial \log \operatorname{det}\left(\prod_{i}\left(S^{i}\right)^{2}\right) \wedge * \omega \\
& +\frac{\sqrt{-1}}{2 \pi} \int_{X} \bar{\partial} \partial \log \operatorname{det}\left(\prod_{i}\left(S^{i}\right)^{-2} h\right) \wedge * \omega
\end{aligned}
$$

By the Poincaré-Lelong formula ([SABK], Ch.II, Section 1, Theorem 2), we have

$$
d_{0}\left(E, K_{0}\right)=\operatorname{par} \operatorname{deg} E+\frac{\sqrt{-1}}{2 \pi} \int_{X} \bar{\partial} \partial \log \operatorname{det}\left(\prod_{i}\left(S^{i}\right)^{-2} h\right) \wedge * \omega
$$

By the construction of the metrics $K_{0}$ and $K_{1}$, it is not difficult to see that $\log \operatorname{det}\left(\prod_{i}\left(S^{i}\right)^{-2} h\right)$ can be extended smoothly to $\bar{X}$, so the last term on the right hand side of the identity vanishes, this proves the proposition.

It is clear that

$$
\begin{aligned}
\left.\int_{X} \operatorname{tr} F_{K_{1}} \wedge \frac{\left(\sqrt{-1}\left(\frac{2}{2-\alpha}\right) \sum_{i=1}^{m} \partial \bar{\partial}\left|\sigma_{i}\right|^{2-\alpha}+\right.}{} C_{\alpha} \omega\right)^{n-1} \\
(n-1) ! \\
=C_{\alpha}^{n-1} \int_{X} \operatorname{tr} F_{K_{1}} \wedge \frac{\omega^{n-1}}{(n-1) !}
\end{aligned}
$$

and

$$
\begin{aligned}
& \int_{X} \bar{\partial} \partial \log \operatorname{det} h \wedge \frac{\left(\sqrt{-1}\left(\frac{2}{2-\alpha}\right) \sum_{i=1}^{m} \partial \bar{\partial}\left|\sigma_{i}\right|^{2-\alpha}+C_{\alpha} \omega\right)^{n-1}}{(n-1) !} \\
&=C_{\alpha}^{n-1} \int_{X} \bar{\partial} \partial \log \operatorname{det} h \wedge \frac{\omega^{n-1}}{(n-1) !}
\end{aligned}
$$

So, we have the following proposition

Proposition 5.4.

$$
d_{\alpha}\left(E, K_{0}\right)=C_{\alpha}^{n-1} \operatorname{par} \operatorname{deg} E
$$


Then we consider the parabolic degree and the analytic degree of a coherent subsheave of $E$.

Proposition 5.5. Let $V$ be a proper coherent subsheaf of $E$ with quotient torsion free.

1) If $K_{1}$ is a metric on $E$

$$
\operatorname{deg} V=\frac{\sqrt{-1}}{2 \pi} \int_{X} \operatorname{tr}\left(\Lambda F_{\left.K_{1}\right|_{V}}\right) d V
$$

2) If $K_{0}$ is the metric defined in Section 3, we have

$$
\text { par } \operatorname{deg} V=d_{0}\left(V, K_{0}\right) .
$$

Consequently, $|\bar{\partial} \pi|_{K_{0}} \in L^{2}(X)$.

Proof. By a theorem of Hironaka ([H],p.145, Corollary 2), we can find a complex manifold $\widetilde{X}$, which is obtained by successively blowing up complex submanifolds, such that the following holds. Let $q: \widetilde{X} \rightarrow \bar{X}$ be the canonical map and let $\left\{P_{i}^{n_{i}}\right\}(i=1, \cdots, N)$ be the components of the exceptional divisor $\mathrm{S}$; then there exist positive integers $\left\{m_{i}\right\}(i=1, \cdots, N)$ such that the canonical map from $q^{*}(V)$ to $q^{*}(E)$ maps $q^{*}(V)$ isomorphically onto a proper subbundle of $q^{*}(E) \otimes \mathcal{O}\left(-m_{i} P_{i}^{n_{i}}\right)$.

We first prove 1), i.e.,

$$
\operatorname{deg}(V)=\frac{\sqrt{-1}}{2 \pi} \int_{X} \operatorname{tr}\left(F_{\left.K_{1}\right|_{V}}\right) \wedge * \omega
$$

Note that for any choice of a metric $\gamma$ on $q^{*}(V)$ we have

$$
\operatorname{deg}(V)=\frac{\sqrt{-1}}{2 \pi} \int_{\widetilde{X}} \operatorname{tr}\left(F_{\gamma}\right) \wedge * q^{*}(\omega)
$$

Now consider the metric on $q^{*}(V)$ obtained from the metric on $q^{*}(E) \otimes$ $\mathcal{O}\left(-m_{i} P_{i}^{n_{i}}\right)$, where we use on $q^{*}(E)$ pullback of $K_{1}$ and on each $\mathcal{O}\left(P_{i}^{n_{i}}\right)$ some metric. Let $\left|S_{i}\right|$ be the norm of the canonical section of $\mathcal{O}\left(P_{i}^{n_{i}}\right)$ with 
respect to the metric. we have

$$
\begin{aligned}
\operatorname{deg} V= & \frac{\sqrt{-1}}{2 \pi} \int_{\tilde{X}} \operatorname{tr}\left(F_{\gamma}\right) \wedge * q^{*}(\omega) \\
= & \frac{\sqrt{-1}}{2 \pi} \int_{\tilde{X} \backslash S} \operatorname{tr}\left(q^{*}\left(F_{\left.K_{1}\right|_{V}}\right)\right) \wedge * q^{*}(\omega) \\
& +\frac{\sqrt{-1}}{2 \pi} \int_{\tilde{X} \backslash S}-\frac{m_{i}}{2} \bar{\partial} \partial \log \left|S_{i}\right|^{2} \wedge * q^{*}(\omega) \\
= & \frac{\sqrt{-1}}{2 \pi} \int_{X} \operatorname{tr}\left(F_{\left.K_{1}\right|_{V}}\right) \wedge * \omega+\frac{\sqrt{-1}}{2 \pi} \int_{\tilde{X} \backslash S}-\frac{m_{i}}{2} \bar{\partial} \partial \log \left|S_{i}\right|^{2} \wedge * q^{*}(\omega) \\
= & \frac{\sqrt{-1}}{2 \pi} \int_{X} \operatorname{tr}\left(F_{\left.K_{1}\right|_{V}}\right) \wedge * \omega-\frac{m_{i}}{2} \int_{\tilde{X}} C_{1}\left(\mathcal{O}\left(P_{i}^{n_{i}}\right)\right) \wedge * q^{*}(\omega) \\
= & \frac{\sqrt{-1}}{2 \pi} \int_{X} \operatorname{tr}\left(F_{\left.K_{1}\right|_{V}}\right) \wedge * \omega
\end{aligned}
$$

where $C_{1}\left(\mathcal{O}\left(P_{i}^{n_{i}}\right)\right)$ is the first Chern form of $\mathcal{O}\left(P_{i}^{n_{i}}\right)$.

From now on, we suppose that $K_{1}$ is the metric of $E$ defined in this section.

It is clear that

$$
\operatorname{tr} F_{\left.K_{0}\right|_{V}}=\operatorname{tr} F_{\left.K_{1}\right|_{V}}+\bar{\partial} \partial \log \operatorname{det}\left(\left(K_{1} \mid V\right)^{-1}\left(\left.K_{0}\right|_{V}\right)\right)
$$

Using 1) we have

$$
d_{0}\left(V, K_{0}\right)=\operatorname{deg} V+\frac{\sqrt{-1}}{2 \pi} \int_{X} \bar{\partial} \partial \log \operatorname{det}\left(\left(\left.K_{1}\right|_{V}\right)^{-1}\left(\left.K_{0}\right|_{V}\right)\right) \wedge * \omega
$$

We will use blow up as above to calculate the second term on the right hand side of the last identity, which equals

$$
\frac{\sqrt{-1}}{2 \pi} \int_{\tilde{X} \backslash q^{-1}(D)} \bar{\partial} \partial \log \operatorname{det}\left(\left(\left.q^{*}\left(K_{1}\right)\right|_{q^{*}(V)}\right)^{-1}\left(\left.q^{*}\left(K_{0}\right)\right|_{q^{*}(V)}\right)\right) \wedge * q^{*}(\omega)
$$

We denote by $D_{i}^{*}$ the proper transform of $D_{i}$, we may assume that $q^{-1}(D)=$ $\sum_{i=1}^{m} D_{i}^{*}+S$ is a divisor with normal crossings.

Suppose that the flag of $\left.V\right|_{D_{i}}$ by coherent subsheaves is

$$
\left.V\right|_{D_{i}}=F_{1}^{i} V \supset \cdots \supset F_{n_{i}}^{i} V \supset\{0\}=F_{n_{i}+1}^{i} V,
$$

the weight attached to the flag is $\alpha_{1}^{i}(V), \cdots, \alpha_{n_{i}}^{i}(V)$ (see Section 2). Let $a=\operatorname{rank} V$. 
Put $\delta_{l}^{i}=\alpha_{j}^{i}$ if $a-r_{j}^{i}<l \leq a-r_{j+1}^{i}$ where $r_{j}^{i}=\operatorname{rankF}_{j}^{i} V, j=1, \cdots, n_{i}$. Set

$$
\begin{aligned}
A^{i} & =\left(\begin{array}{lll}
\left|\sigma_{i}^{*}\right|^{2 \delta_{1}^{i}} & & \\
& \ddots & \\
& & \left|\sigma_{i}^{*}\right|^{2 \delta_{a}^{i}}
\end{array}\right) \\
& B^{i}=\left(\begin{array}{ccc}
\prod_{l=1}^{N}\left|S_{l}\right|^{2 \delta_{1}^{i}} & & \\
& \ddots & \\
& & \prod_{l=1}^{N}\left|S_{l}\right|^{2 \delta_{a}^{i}}
\end{array}\right)
\end{aligned}
$$

where $\sigma_{i}^{*}$ is the canonical section of $D_{i}^{*}$.

We have

$$
\begin{aligned}
& \frac{\sqrt{-1}}{2 \pi} \int_{\tilde{X} \backslash q^{-1}(D)} \bar{\partial} \partial \log \operatorname{det}\left(\left(\left.q^{*}\left(K_{1}\right)\right|_{q^{*}(V)}\right)^{-1}\left(\left.q^{*}\left(K_{0}\right)\right|_{q^{*}(V)}\right)\right) \wedge * q^{*}(\omega) \\
= & \frac{\sqrt{-1}}{2 \pi} \int_{\tilde{X} \backslash q^{-1}(D)} \bar{\partial} \partial \log \operatorname{det}\left(\prod_{i}\left(A^{i} B^{i}\right)^{-1}\left(\left.q^{*}\left(K_{1}\right)\right|_{q^{*}(V)}\right)^{-1}\left(\left.q^{*}\left(K_{0}\right)\right|_{q^{*}(V)}\right)\right) \\
& \wedge * q^{*}(\omega)+\frac{\sqrt{-1}}{2 \pi} \int_{\tilde{X} \backslash q^{-1}(D)} \bar{\partial} \partial \log \operatorname{det}\left(\prod_{i}\left(A^{i} B^{i}\right)\right) \wedge * q^{*}(\omega)
\end{aligned}
$$

Let $p \in q^{-1}(D)$.Assume that $D_{1}^{*}, \cdots, D_{k}^{*}, P_{1}^{n_{1}}, \cdots, P_{b}^{n_{b}}$ pass through $p$. Suppose that $f_{1}, \cdots, f_{a}$ is a holomorphic frame of $q^{*}(V)$ in a neighborhood $U_{p}$ of $\mathrm{p}$ and that $f$ is a holomorphic frame of $\mathcal{O}\left(-m_{i} P_{i}^{n_{i}}\right)$, assume that $f_{i}=g_{i j} q^{*}\left(e_{j}\right) \otimes f$ where $e_{1}, \cdots, e_{r}$ is a holomorphic basis of $E$ around $q(p)$ preserving the flags, $g=\left(g_{i j}\right)$ is a matrix with constant rank $a$. Furthermore, we may assume that $f_{i}=q^{*}\left(e_{k_{i}}\right) \otimes f$ on $D_{p}^{*}=\cup_{j=1}^{k} D_{j}^{*}, i=1, \cdots, a$, that is $\left.g_{i j}\right|_{D_{p}^{*}}=0$ if $j \neq k_{i}$ and $\left.g_{i k_{i}}\right|_{D_{p}^{*}}=1$. We have

$$
\begin{aligned}
\left\langle f_{i}, f_{j}\right\rangle_{H^{\prime}} & =g_{i l} \bar{g}_{j l} \\
\left\langle f_{i}, f_{j}\right\rangle_{q^{*}(H)} & =\prod_{t=1}^{k}\left|\sigma_{t}^{*}\right|^{2 \beta_{l}^{t}} \prod_{t_{1}=1}^{b}\left|S_{t_{1}}\right|^{2 \beta_{l}^{t}} g_{i l} \bar{g}_{j l}
\end{aligned}
$$

Here $H$ is the local metric on $E^{\prime}$ defined in Section $3, H^{\prime}$ is the local metric on $E$ constructed in this section, $\beta_{l}^{t}$ is defined in Section 3.

Let $\left(z_{1}, \cdots, z_{n}\right)$ be a local coordinate system around $p$ and $P_{i}^{n_{i}}$ is defined by $z_{i}=0, i=1, \cdots, b$. Then

$$
q^{*}(\omega)=\sum_{l, m}\left(\prod_{i=1, i \neq l}^{b} z_{i} \prod_{j=1, j \neq m}^{b} \bar{z}_{j} \omega_{l \bar{m}} d z_{l} \wedge d \bar{z}_{m}\right)
$$


By (5),(6) and (7) we can see that

$$
\begin{gathered}
\int_{\tilde{X} \backslash q^{-1}(D)} \bar{\partial} \partial \log \operatorname{det}\left(\prod_{i}\left(A^{i} B^{i}\right)^{-1}\left(\left.q^{*}\left(K_{1}\right)\right|_{q^{*}(V)}\right)^{-1}\left(\left.q^{*}\left(K_{0}\right)\right|_{q^{*}(V)}\right)\right) \wedge * q^{*}(\omega) \\
=0
\end{gathered}
$$

We therefore have

$$
\begin{aligned}
& \frac{\sqrt{-1}}{2 \pi} \int_{\tilde{X} \backslash q^{-1}(D)} \bar{\partial} \partial \log \operatorname{det}\left(\left(\left.q^{*}\left(K_{1}\right)\right|_{q^{*}(V)}\right)^{-1}\left(\left.q^{*}\left(K_{0}\right)\right|_{q^{*}(V)}\right)\right) \wedge * q^{*}(\omega) \\
&=\sum_{i=1}^{m} \sum_{j=1}^{a} \delta_{j}^{i}\left(\frac{\sqrt{-1}}{2 \pi} \int_{\tilde{X} \backslash q^{-1}(D)} \bar{\partial} \partial \log \left|\sigma_{i}^{*}\right|^{2} \wedge * q^{*}(\omega)\right) \\
&+\sum_{i=1}^{m} \sum_{j=1}^{a} \delta_{j}^{i} \sum_{l=1}^{N} \frac{\sqrt{-1}}{2 \pi} \int_{\tilde{X} \backslash q^{-1}(D)} \bar{\partial} \partial \log \left|S_{l}\right|^{2} \wedge * q^{*}(\omega) \\
&= \sum_{i=1}^{m} \sum_{j=1}^{a} \delta_{j}^{i}\left(\frac{\sqrt{-1}}{2 \pi} \int_{\tilde{X} \backslash D} \bar{\partial} \partial \log \left|\sigma_{i}^{*}\right|^{2} \wedge * q^{*}(\omega)\right) \\
&= \sum_{i=1}^{m} \sum_{j=1}^{a} \delta_{j}^{i} \operatorname{deg}\left[D_{i}\right]
\end{aligned}
$$

So

$$
d_{0}\left(V, K_{0}\right)=\operatorname{par} \operatorname{deg} V
$$

This completes the proof of the proposition.

Similarly we also have

Proposition 5.6. Let $V$ be a proper coherent subsheaf of $E$ with quotient torsion free.

$$
d_{\alpha}\left(V, K_{0}\right)=C_{\alpha}^{n-1} \operatorname{par} \operatorname{deg} V
$$

Proposition 5.7. Suppose that $K_{0}$ is the metric constructed in Section 3. If $K$ and $K_{0}$ are compatible with respect to $\omega_{\alpha}$, then $d_{\alpha}(V, K)=d_{\alpha}\left(V, K_{0}\right)$.

Proof. Let $h=K_{0}^{-1} K$. We adopt the notations in the proof of Proposition 5.5. In particular $q: \widetilde{X} \in \bar{X}$ is the blow up. We shall prove

$$
\begin{gathered}
\int_{\tilde{X} \backslash q^{-1}(D)} \bar{\partial} \partial \log \operatorname{det}\left(\prod_{i}\left(A^{i} B^{i}\right)^{-1}\left(\left.q^{*}\left(K_{1}\right)\right|_{q^{*}(V)}\right)^{-1}\left(\left.q^{*}(K)\right|_{q^{*}(V)}\right)\right) \wedge * q^{*}\left(\omega_{\alpha}\right) \\
=0
\end{gathered}
$$


For any point $p \in q^{-1}(D)$, around it we use the holomorphic frame $e_{i}^{(1, \cdots, k)}$ defined in Section 3.

Suppose that

$$
q^{*}(h) q^{*}\left(e_{i}^{(1, \cdots, k)}\right)=h_{i j} q^{*}\left(e_{j}^{(1, \cdots, k)}\right)
$$

and

$$
f_{i}=g_{i j}^{\prime} q^{*}\left(e_{j}^{(1, \cdots, k)}\right) \otimes f
$$

It is clear that

$$
g_{i j}=g_{i j}^{\prime} q^{*}\left(\prod_{l=1}^{k} \sigma_{l}^{-\alpha_{k_{l}}^{l}}\right) \text { if } r k\left(F_{k_{l}+1}^{l}\right)<j \leq r k\left(F_{k_{l}}^{l}\right)
$$

where $k_{l}=1, \cdots, m_{l}$.

We have

$$
\left\langle q^{*}(h) f_{i}, f_{j}\right\rangle_{H}=g_{i l}^{\prime} h_{l \delta} \overline{g_{j \delta}^{\prime}}\langle f, f\rangle
$$

Here $H$ is the local metric on $E^{\prime}$ defined in Section 3.

Since $q^{*} \sigma_{i}=\sigma_{i}^{*} \prod_{t_{1}=1}^{b} S_{t_{1}},|h|_{K_{0}} \in L^{\infty}(X),|\bar{\partial} h|_{K_{0}, \omega_{\alpha}} \in L^{2}\left(X, \omega_{\alpha}\right)$, using (6) we can show the claim, which yields the proposition.

Finally, we consider the equivalence between parabolic stability and analytic stability.

The following lemma is a corollary of a theorem of Siu ([Siu], Theorem $4.5)$.

Lemma 5.8. Suppose that $A$ is a thick set in $P^{*}\left(k_{0}, n-1\right)\left(0 \leq k_{0} \leq n-\right.$ $1)$, assume that $\mathcal{G}$ is a coherent analytic sheaf on $P^{*}\left(k_{0}, n-1\right) \times \triangle$ and that $\mathcal{F}$ is a coherent analytic subsheaf of $\mathcal{G}$ with quotient torsion free on $P^{*}\left(k_{0}, n-1\right) \times \Delta^{*}$, where $\Delta=\{|z|<1\}, \Delta^{*}=\Delta \backslash\{0\}$. If for every point $p \in A,\left.\mathcal{F}\right|_{\{p\} \times \Delta^{*}}$ can be extended to $\{p\} \times \triangle$ as a coherent analytic subsheaf of $\left.\mathcal{G}\right|_{\{p\} \times \triangle}$, then $\mathcal{F}$ can be extended uniquely to a coherent analytic subsheaf of $\mathcal{G}$ on $P^{*}\left(k_{0}, n-1\right) \times \triangle$.

Li-Narasimhan showed in [L-N] (Lemma 6.2) that the extension of a coherent subsheaf of $E^{\prime}=\left.E\right|_{X}$ is a local problem. So applying Lemma 10.6 in [S1] and using Lemma 5.8 at most $n$ times, we can prove the following proposition.

Proposition 5.9. Suppose that $K_{0}$ is the metric on $E^{\prime}=\left.E\right|_{X}$ constructed in Section 3. If $V$ is a proper coherent subsheaf of $E^{\prime}$ with quotient torsion free and $\left|\bar{\partial} \pi_{V}\right|_{K_{0}} \in L^{2}(X)$, then it extends to a coherent subsheaf of $E$. 
Proposition 5.10. Suppose that $E$ is a parabolic bundle, assume that $K_{0}$ is the metric constructed in Section 3. Then $E$ is parabolic stable if and only if $\left(E, K_{0}\right)$ is analytic stable with respect to $\omega_{\alpha}$.

Proof. Using (4) we can obtain

$$
\int_{X}\left|\bar{\partial} \pi_{V}\right|_{K_{0}} d V \leq C_{\alpha} \int_{X}\left|\bar{\partial} \pi_{V}\right|_{K_{0}, \omega_{\alpha}} d V_{\alpha}
$$

So the proposition follows from Proposition 5.4, Proposition 5.6 and Proposition 5.9.

\section{The existence of H-E metrics .}

In this section we prove one of our main theorems in this paper.

Definition 6.1. A Hermitian metric $H$ on $E^{\prime}=\left.E\right|_{X}$ is called HermitianEinstein with respect to $\omega_{\alpha}$, if $\Lambda_{\alpha} F_{H}^{\perp}=0$ where $F_{H}^{\perp}=F_{H}-\frac{\operatorname{tr} F_{H}}{\operatorname{rankE} I} I$ is the trace free part of the curvature $F_{H}, I$ is the identity endomorphism of $E^{\prime}$.

Definition 6.2. Suppose that $E$ is a parabolic bundle, $K$ is a Hermitian metric on $E^{\prime}=\left.E\right|_{X}$, we say that it is compatible with the parabolic structure with respect to $\omega_{\alpha}$ if $K$ and $K_{0}$ are compatible with respect to $\omega_{\alpha}$, where $K_{0}$ is defined in Section 3.

We set

$$
\gamma_{0}=\min \left\{\alpha_{l}^{i}-\alpha_{l-1}^{i}, 1-\left(\alpha_{l}^{i}-\alpha_{l-1}^{i}\right) \mid l=2, \cdots, m_{i}+1, i=1, \cdots, m\right\} .
$$

Theorem 6.3. Let $\bar{X}$ be a compact Kähler manifold of complex dimension $n$ and $D$ a divisor of $\bar{X}$ with normal crossings. Let $E$ be a holomorphic vector bundle with a parabolic structure along $D$. If $E$ is parabolic stable there exists a Hermitian-Einstein metric with respect to $\omega_{\alpha}$ for any $2\left(1-\gamma_{0}\right) \leq \alpha<2$ on $E^{\prime}$ compatible with the parabolic structure with respect to $\omega_{\alpha}$. Conversely, if $E$ is indecomposable and $E^{\prime}$ admits a Hermitian-Einstein metric with respect to $\omega_{\alpha}(0 \leq \alpha<2)$ compatible with the parabolic structure with respect to $\omega_{\alpha}$, then $E$ is parabolic stable.

Proof. If $E$ is parabolic stable, by Proposition 5.10 we know that $\left(E, K_{0}\right)$ is analytic stable with respect to $\omega_{\alpha}$ for any $0 \leq \alpha<2$. According to (3) 
we know that we can choose any $2\left(1-\gamma_{0}\right) \leq \alpha<2$ such that $\left|\Lambda_{\alpha} F_{K_{0}}\right|_{K_{0}} \in$ $L^{\infty}(X)$. Theorem 1 in [S1] yields that there is a H-E metric on $E^{\prime}$ compatible with the parabolic structure with respect to $\omega_{\alpha}$.

Conversely, suppose that $H$ is a H-E metric compatible with the parabolic structure with respect to $\omega_{\alpha}$, we have par $\operatorname{deg} E=C_{\alpha}^{1-n} d_{\alpha}(E, H)$. Suppose that $V$ is a proper coherent subsheaf of $E$ with quotient torsion free, by Proposition 5.6 and Proposition 5.7 we have $\operatorname{par} \operatorname{deg} V=C_{\alpha}^{1-n} d_{\alpha}(V, H)$. Then by an argument similar to the one used in the proof of Theorem 7.3 in [L-N] we can show that $E$ is parabolic stable if $E$ is indecomposable.

\section{Chern number inequality(I).}

Suppose that $H$ is a Hermitian-Einstein metric on $E^{\prime}$ compatible with the parabolic structure with respect to $\omega_{\alpha}\left(2\left(1-\gamma_{0}\right) \leq \alpha<2\right)$, which is obtained in Theorem 6.3. It was proved in [S1] (Proposition 3.4) that

$$
\left(2 C_{2}(E, H)-\frac{r-1}{r} C_{1}(E, H)^{2}\right)\left[\omega_{\alpha}\right]^{n-2} \geq 0
$$

where $r=\operatorname{rankE}$.

$$
\begin{aligned}
& C_{1}(E, H)=\frac{\sqrt{-1}}{2 \pi} \operatorname{tr} F_{H}, \\
& C_{2}(E, H)=-\frac{1}{8 \pi^{2}}\left(\operatorname{tr} F_{H} \wedge \operatorname{tr} F_{H}-\operatorname{tr} F_{H} \wedge F_{H}\right)
\end{aligned}
$$

Since $\operatorname{det} H=\operatorname{det} K_{0}$, we have $C_{1}(E, H)=C_{1}\left(E, K_{0}\right)$.

Lemma 7.1.

$$
\int_{X} C_{2}(E, H) \wedge \omega_{\alpha}^{n-2} \leq \int_{X} C_{2}\left(E, K_{0}\right) \wedge \omega_{\alpha}^{n-2}
$$

Proof. It suffices to show that

$$
\int_{X} \operatorname{tr}\left(F_{H} \wedge F_{H}\right) \wedge \omega_{\alpha}^{n-2} \leq \int_{X} \operatorname{tr}\left(F_{K_{0}} \wedge F_{K_{0}}\right) \wedge \omega_{\alpha}^{n-2}
$$

Suppose that $f$ is a compactly suppored function on $X$, and we set $v=$ $-4 \pi \sqrt{-1} \partial \bar{\partial} f$. Simpson [S1] showed that

$$
\begin{array}{rl}
\int_{X} & f\left(\operatorname{tr}\left(F_{K_{0}} \wedge F_{K_{0}}\right)-\operatorname{tr}\left(F_{H} \wedge F_{H}\right)\right) \wedge \omega_{\alpha}^{n-2} \\
\quad= & \frac{\sqrt{-1}}{2 \pi} \int_{X} \operatorname{tr}\left(s F_{K_{0}}\right) v \wedge \omega_{\alpha}^{n-2}-\frac{\sqrt{-1}}{2 \pi} \int_{X} \operatorname{tr}\left(\Psi(s)(\bar{\partial} s) \partial_{K_{0}} s\right) v \wedge \omega_{\alpha}^{n-2}
\end{array}
$$


where $e^{s}=K_{0}^{-1} H, \Psi(s)$ is constructed as in the definition of Donaldson's functional (see [S1] Section 5).

We choose

$$
f_{\beta}=\max \left\{0,1+\frac{\log |\sigma|^{2}}{\beta}\right\}
$$

Set $X_{\beta}=\left\{\left.x \in X|\log | \sigma\right|^{2}>-\beta\right\}$. We now recall how one gets $H$ from $K_{0}$ (see [S1] Section 6 and Section 7).

One solves the heat equation

$$
\left\{\begin{array}{l}
H^{-1} \frac{d H}{d t}=-\sqrt{-1} \Lambda_{\alpha} F_{H}^{\perp} \\
\left.H\right|_{t=0}=K_{0} \\
\operatorname{det} H=\operatorname{det} K_{0}
\end{array}\right.
$$

on $X_{\beta}$ with Dirichlet boundary condition $\left.H\right|_{\partial X_{\beta}}=K_{0}$. If the solution is denoted by $H_{\beta}(t)$, one shows that $H_{\beta}(t) \rightarrow H(t)$ in $C^{1,0}$ over compact sets in $X$, as $\beta \rightarrow \infty$. $H(t)$ is a solution of the heat equation, and there exists a subsequence $t_{i} \rightarrow \infty$ such that $H\left(t_{i}\right) \rightarrow H$ weakly in $L_{2, l o c}^{p}$.

Set $e^{s_{\beta}}=h_{\beta}=K_{0}^{-1} H_{\beta}(t)$ in $X_{\beta}$ and $s_{\beta}=0$ outside $X_{\beta}$. Since ([S1], Lemma $3.1(\mathrm{c}))$

$$
\triangle_{\alpha} \operatorname{trh} h_{\beta}=-\sqrt{-1} \operatorname{tr}\left(h_{\beta}\left(\Lambda_{\alpha} F_{H_{\beta}}^{\perp}-\Lambda_{\alpha} F_{K_{0}}^{\perp}\right)\right)+\left|\left(\bar{\partial} h_{\beta}\right) h_{\beta}^{-\frac{1}{2}}\right|_{K_{0}, \omega_{\alpha}}^{2}
$$

and $\left.\frac{\partial}{\partial n} \operatorname{trh}_{\beta}\right|_{\partial X_{\beta}} \leq 0$, because $t r h_{\beta} \geq r=\left.t r h_{\beta}\right|_{\partial X_{\beta}}$, where $\triangle_{\alpha}$ is the Laplace operator with respect to $\omega_{\alpha}$, we have

$$
\int_{X}\left|\left(\bar{\partial} h_{\beta}\right) h_{\beta}^{-\frac{1}{2}}\right|_{K_{0}, \omega_{\alpha}}^{2} d V_{\alpha} \leq C .
$$

Here $C$ is a positive constant independent of $\beta$.

By Proposition 5.3 and Lemma 7.1 in [S1] we can see that $\left|h_{\beta}\right|_{K_{0}}$ is bounded on both side. So

$$
\int_{X}\left|\bar{\partial} s_{\beta}\right|_{K_{0}, \omega_{\alpha}}^{2} d V_{\alpha} \leq C
$$


We have

$$
\begin{aligned}
& \int_{X_{\beta}} f_{\beta} \operatorname{tr}\left(F_{H_{\beta}} \wedge F_{H_{\beta}}\right) \wedge \omega_{\alpha}^{n-2} \\
&= \int_{X_{\beta}} f_{\beta} \operatorname{tr}\left(F_{K_{0}} \wedge F_{K_{0}}\right) \wedge \omega_{\alpha}^{n-2}-\frac{\sqrt{-1}}{2 \pi} \int_{X_{\beta}} \operatorname{tr}\left(s_{\beta} F_{K_{0}}\right) v \wedge \omega_{\alpha}^{n-2} \\
& \quad+\frac{\sqrt{-1}}{2 \pi} \int_{X_{\beta}} \operatorname{tr}\left(\Psi\left(s_{\beta}\right)\left(\bar{\partial} s_{\beta}\right) \partial_{K_{0}} s_{\beta}\right) v \wedge \omega_{\alpha}^{n-2}
\end{aligned}
$$

Note that $\left.s_{\beta}\right|_{\partial X_{\beta}}=0,\left.f_{\beta}\right|_{\partial X_{\beta}}=0$, we have

$$
\left.\operatorname{tr}\left(s_{\beta} F_{K_{0}}\right) v\right|_{\partial X_{\beta}}=0
$$

and

$$
\left.\operatorname{tr}\left(\Psi\left(s_{\beta}\right)\left(\bar{\partial} s_{\beta}\right) \partial_{K_{0}} s_{\beta}\right) v\right|_{\partial X_{\beta}}=0
$$

In $X_{\beta}$,

$$
v=-4 \pi \sqrt{-1} \partial \bar{\partial} f_{\beta}=-\frac{4 \pi \sqrt{-1}}{\beta} \partial \bar{\partial} \log |\sigma|^{2}
$$

By Poincaré-Lelong formula, we have

$$
\begin{aligned}
\int_{X_{\beta}} f_{\beta} \operatorname{tr}\left(F_{H_{\beta}} \wedge F_{H_{\beta}}\right) \wedge & \omega_{\alpha}^{n-2} \\
\leq & \int_{X_{\beta}} f_{\beta} \operatorname{tr}\left(F_{K_{0}} \wedge F_{K_{0}}\right) \wedge \omega_{\alpha}^{n-2} \\
& +\frac{C}{\beta} \int_{X_{\beta}}\left|\operatorname{tr} \Lambda_{\alpha} F_{K_{0}}\right| d V_{\alpha}+\frac{C}{\beta} \int_{X_{\beta}}\left|\bar{\partial} s_{\beta}\right|_{K_{0}, \omega_{\alpha}}^{2} d V_{\alpha}
\end{aligned}
$$

By the Riemman bilinear relations, one gets

$$
\operatorname{tr}\left(F_{H_{\beta}} \wedge F_{H_{\beta}}\right) \wedge \omega_{\alpha}^{n-2} \geq-C\left|\Lambda_{\alpha} F_{H_{\beta}}\right|^{2} \omega_{\alpha}^{n}
$$

Since

$$
\sup _{X_{\beta}}\left|\Lambda_{\alpha} F_{H_{\beta}}^{\perp}\right| \leq \sup _{X_{\beta}}\left|\Lambda_{\alpha} F_{K_{0}}^{\perp}\right| \leq C
$$

and $\operatorname{tr} F_{H_{\beta}}=\operatorname{tr} F_{K_{0}}$, we have

$$
\operatorname{tr}\left(F_{H_{\beta}} \wedge F_{H_{\beta}}\right) \wedge \omega_{\alpha}^{n-2} \geq-C \omega_{\alpha}^{n}
$$

Letting $\beta \rightarrow \infty$, using Fatou's lemma we obtain

$$
\int_{X} \operatorname{tr}\left(F_{H(t)} \wedge F_{H(t)}\right) \wedge \omega_{\alpha}^{n-2} \leq \int_{X} \operatorname{tr}\left(F_{K_{0}} \wedge F_{K_{0}}\right) \wedge \omega_{\alpha}^{n-2}
$$


Applying Fatou's lemma again, we get

$$
\int_{X} \operatorname{tr}\left(F_{H} \wedge F_{H}\right) \wedge \omega_{\alpha}^{n-2} \leq \int_{X} \operatorname{tr}\left(F_{K_{0}} \wedge F_{K_{0}}\right) \wedge \omega_{\alpha}^{n-2}
$$

Proposition 7.2. Let $\bar{X}$ be a compact Kähler manifold of complex dimension $n$ and $D$ a divisor of $\bar{X}$ with normal crossings. Let $E$ be a holomorphic vector bundle over $\bar{X}$ with a parabolic structure along $D$. Let $K_{0}$ be the metric of $E^{\prime}$ constructed in Section 3. If $E$ is parabolic stable, for any $2\left(1-\gamma_{0}\right) \leq \alpha<2$ such that

$$
\left(2 C_{2}\left(E, K_{0}\right)-\frac{r-1}{r} C_{1}\left(E, K_{0}\right)^{2}\right)\left[\omega_{\alpha}\right]^{n-2} \geq 0
$$

where $r=\operatorname{rankE}$.

The proposition follows from Lemma 7.1 and (8).

\section{Lemma 7.3.}

$$
\begin{aligned}
& C_{\alpha}^{2-n} \int_{X} C_{1}\left(E, K_{0}\right) \wedge C_{1}\left(E, K_{0}\right) \wedge \omega_{\alpha}^{n-2} \\
& =\int_{X} C_{1}(E) \wedge C_{1}(E) \wedge \omega^{n-2} \\
& \quad+2 \sum_{i=1}^{m} \sum_{l=1}^{m_{i}} \alpha_{l}^{i} \operatorname{rank}\left(F_{l}^{i} / F_{l+1}^{i}\right) \operatorname{deg}\left(\left.E\right|_{D_{i}}\right) \\
& \quad+\sum_{i, j=1}^{m}\left(\left(\sum_{l=1}^{m_{i}} \alpha_{l}^{i} \operatorname{rank}\left(F_{l}^{i} / F_{l+1}^{i}\right)\right)\left(\sum_{l=1}^{m_{j}} \alpha_{l}^{j} \operatorname{rank}\left(F_{l}^{j} / F_{l+1}^{j}\right)\right) D_{i} \cdot D_{j}\right)
\end{aligned}
$$

where $D_{i} \cdot D_{j}=\int_{X} C_{1}\left(\left[D_{i}\right]\right) \wedge C_{1}\left(\left[D_{j}\right]\right) \wedge \omega^{n-2}$ is the intersection number of $D_{i}$ and $D_{j}(i, j=1, \cdots, m)$.

Proof. Suppose that $K_{1}$ is the metric constructed in Section 5. Set $h=$ $K_{1}^{-1} K_{0}$, then $\operatorname{tr} F_{K_{0}}=\operatorname{tr} F_{K_{1}}+\bar{\partial} \partial \log \operatorname{det} h$. So,

$$
\begin{aligned}
C_{1}\left(E, K_{0}\right) \wedge & C_{1}\left(E, K_{0}\right) \\
= & \left(\frac{\sqrt{-1}}{2 \pi}\right)^{2}\left(\operatorname{tr} F_{K_{1}}+\bar{\partial} \partial \log \operatorname{det} h\right) \wedge\left(\operatorname{tr} F_{K_{1}}+\bar{\partial} \partial \log \operatorname{det} h\right) \\
= & \left(\frac{\sqrt{-1}}{2 \pi}\right)^{2}\left(\left(t r F_{K_{1}}\right)^{2}+2 \operatorname{tr} F_{K_{1}} \wedge \bar{\partial} \partial \log \operatorname{det} h\right. \\
& +\bar{\partial} \partial \log \operatorname{det} h \wedge \bar{\partial} \partial \log \operatorname{det} h)
\end{aligned}
$$


We adopt the same notations as that in the proof of Proposition 5.3, we have

$$
\begin{aligned}
& \int_{X} C_{1}\left(E, K_{0}\right) \wedge C_{1}\left(E, K_{0}\right) \wedge \omega_{\alpha}^{n-2} \\
& =\left(\frac{\sqrt{-1}}{2 \pi}\right)^{2} \int_{X}\left(t F_{K_{1}}\right)^{2} \wedge \omega_{\alpha}^{n-2} \\
& +2\left(\frac{\sqrt{-1}}{2 \pi}\right)^{2} \int_{X} \operatorname{tr} F_{K_{1}} \wedge \bar{\partial} \partial \log \operatorname{det}\left(\Pi_{i}\left(S^{i}\right)^{2}\right) \wedge \omega_{\alpha}^{n-2} \\
& +2\left(\frac{\sqrt{-1}}{2 \pi}\right)^{2} \int_{X} \operatorname{tr} F_{K_{1}} \wedge \bar{\partial} \partial \log \operatorname{det}\left(\Pi_{i}\left(S^{i}\right)^{-2} h\right) \wedge \omega_{\alpha}^{n-2} \\
& +\left(\frac{\sqrt{-1}}{2 \pi}\right)^{2} \int_{X} \bar{\partial} \partial \log \operatorname{det}\left(\Pi_{i}\left(S^{i}\right)^{2}\right) \wedge \bar{\partial} \partial \log \operatorname{det}\left(\Pi_{i}\left(S^{i}\right)^{2}\right) \wedge \omega_{\alpha}^{n-2} \\
& +\left(\frac{\sqrt{-1}}{2 \pi}\right)^{2} \int_{X} \bar{\partial} \partial \log \operatorname{det}\left(\Pi_{i}\left(S^{i}\right)^{-2} h\right) \wedge \bar{\partial} \partial \log \operatorname{det}\left(\Pi_{i}\left(S^{i}\right)^{-2} h\right) \wedge \omega_{\alpha}^{n-2} \\
& +2\left(\frac{\sqrt{-1}}{2 \pi}\right)^{2} \int_{X} \bar{\partial} \partial \log \operatorname{det}\left(\Pi_{i}\left(S^{i}\right)^{2}\right) \wedge \bar{\partial} \partial \log \operatorname{det}\left(\Pi_{i}\left(S^{i}\right)^{-2} h\right) \wedge \omega_{\alpha}^{n-2}
\end{aligned}
$$

By the construction of the metrics $K_{1}$ and $K_{0}$, it is not difficult to see that $\log \operatorname{det}\left(\Pi_{i}\left(S^{i}\right)^{-2} h\right)$ can be extended smoothly to $\bar{X}$, so

$$
\begin{aligned}
\int_{X} t r F_{K_{1}} \wedge & \bar{\partial} \partial \log \operatorname{det}\left(\Pi_{i}\left(S^{i}\right)^{-2} h\right) \wedge \omega_{\alpha}^{n-2} \\
& =\int_{X} \bar{\partial} \partial \log \operatorname{det}\left(\Pi_{i}\left(S^{i}\right)^{-2} h\right) \wedge \bar{\partial} \partial \log \operatorname{det}\left(\Pi_{i}\left(S^{i}\right)^{-2} h\right) \wedge \omega_{\alpha}^{n-2} \\
& =\int_{X} \bar{\partial} \partial \log \operatorname{det}\left(\Pi_{i}\left(S^{i}\right)^{2}\right) \wedge \bar{\partial} \partial \log \operatorname{det}\left(\Pi_{i}\left(S^{i}\right)^{-2} h\right) \wedge \omega_{\alpha}^{n-2} \\
& =0
\end{aligned}
$$

Thus the Poincaré-Lelong formula yields the lemma. 


\section{Lemma 7.4.}

$$
\begin{aligned}
C_{\alpha}^{2-n}\left(\frac{\sqrt{-1}}{2 \pi}\right)^{2} \int_{X} & \operatorname{tr}\left(F_{K_{0}} \wedge F_{K_{0}}\right) \wedge \omega_{\alpha}^{n-2} \\
= & \left(\frac{\sqrt{-1}}{2 \pi}\right)^{2} \int_{X} \operatorname{tr}\left(F_{K_{1}} \wedge F_{K_{1}}\right) \wedge \omega^{n-2} \\
& +2 \sum_{i=1}^{m} \sum_{l=1}^{m_{i}} \alpha_{l}^{i} \operatorname{deg}\left(F_{l}^{i} / F_{l+1}^{i}\right) \\
& +\sum_{i=1}^{m} \sum_{l=1}^{m_{i}}\left(\alpha_{l}^{i}\right)^{2} \operatorname{rank}\left(F_{l}^{i} / F_{l+1}^{i}\right) D_{i}^{2}
\end{aligned}
$$

where $D_{i}^{2}=\int_{X} C_{1}\left[D_{i}\right] \wedge C_{1}\left[D_{i}\right] \wedge \omega^{n-2}$ is the self-intersection number of $D_{i},(i=1, \cdots, m)$.

Proof. It is clear that

$$
F_{K_{0}}=F_{K_{1}}+\bar{\partial}\left(h^{-1} \partial_{K_{1}} h\right)
$$

where $h=K_{1}^{-1} K_{0}$. So,

$F_{K_{0}} \wedge F_{K_{0}}=F_{K_{1}} \wedge F_{K_{1}}+2 F_{K_{1}} \wedge \bar{\partial}\left(h^{-1} \partial_{K_{1}} h\right)+\bar{\partial}\left(h^{-1} \partial_{K_{1}} h\right) \wedge \bar{\partial}\left(h^{-1} \partial_{K_{1}} h\right)$

Note that

$$
\bar{\partial}\left(h^{-1} \partial_{K_{1}} h\right)=\bar{\partial}\left((S h)^{-1} \partial_{K_{1}}(S h)\right)-\bar{\partial}\left(h^{-1}\left(S^{-1} \partial_{K_{1}} S\right) h\right)
$$

where $S=\Pi_{i}\left(S^{i}\right)^{-2}$. By the construction of the metrics $K_{1}$ and $K_{0}$ we know that $(S h)$ can be seen as an endomorphism of $E$, we have

$$
\begin{aligned}
& \left(\frac{\sqrt{-1}}{2 \pi}\right)^{2} \int_{X} \operatorname{tr}\left(F_{K_{0}} \wedge F_{K_{0}}\right) \wedge \omega_{\alpha}^{n-2} \\
& =\left(\frac{\sqrt{-1}}{2 \pi}\right)^{2} \int_{X} \operatorname{tr}\left(F_{K_{1}} \wedge F_{K_{1}}\right) \wedge \omega_{\alpha}^{n-2} \\
& \quad-2\left(\frac{\sqrt{-1}}{2 \pi}\right)^{2} \int_{X} \operatorname{tr}\left(F_{K_{1}} \wedge \bar{\partial}\left(h^{-1}\left(S^{-1} \partial_{K_{1}} S\right) h\right)\right) \wedge \omega_{\alpha}^{n-2} \\
& \quad+\left(\frac{\sqrt{-1}}{2 \pi}\right)^{2} \int_{X} \operatorname{tr}\left(\bar{\partial}\left(h^{-1}\left(S^{-1} \partial_{K_{1}} S\right) h\right) \wedge \bar{\partial}\left(h^{-1}\left(S^{-1} \partial_{K_{1}} S\right) h\right)\right) \wedge \omega_{\alpha}^{n-2}
\end{aligned}
$$


A simple calculation shows that

$$
\begin{aligned}
C_{\alpha}^{2-n}\left(\frac{\sqrt{-1}}{2 \pi}\right)^{2} \int_{X} \operatorname{tr}\left(F_{K_{1}}\right. & \left.\wedge \bar{\partial}\left(h^{-1}\left(S^{-1} \partial_{K_{1}} S\right) h\right)\right) \wedge \omega_{\alpha}^{n-2} \\
& =\left(\frac{\sqrt{-1}}{2 \pi}\right)^{2} \int_{X} \operatorname{tr}\left(F_{K_{1}} \wedge \bar{\partial}\left(S^{-1} \partial_{K_{1}} S\right)\right) \wedge \omega^{n-2} \\
& =-\sum_{i=1}^{m} \sum_{l=1}^{m_{i}} \alpha_{l}^{i} \operatorname{deg}\left(F_{l}^{i} / F_{l+1}^{i}\right)
\end{aligned}
$$

Similarly, we have

$$
\begin{gathered}
C_{\alpha}^{2-n}\left(\frac{\sqrt{-1}}{2 \pi}\right)^{2} \int_{X} \operatorname{tr}\left(\bar{\partial}\left(h^{-1}\left(S^{-1} \partial_{K_{1}} S\right) h\right) \wedge \bar{\partial}\left(h^{-1}\left(S^{-1} \partial_{K_{1}} S\right) h\right)\right) \wedge \omega_{\alpha}^{n-2} \\
=\left(\frac{\sqrt{-1}}{2 \pi}\right)^{2} \int_{X} \operatorname{tr}\left(\bar{\partial}\left(S^{-1} \partial_{K_{1}} S\right) \wedge \bar{\partial}\left(S^{-1} \partial_{K_{1}} S\right)\right) \wedge \omega^{n-2} \\
=\sum_{i=1}^{m} \sum_{l=1}^{m_{i}}\left(\alpha_{l}^{i}\right)^{2} \operatorname{rank}\left(F_{l}^{i} / F_{l+1}^{i}\right) D_{i}^{2}
\end{gathered}
$$

This completes the proof of the lemma.

Theorem 7.5. Let $\bar{X}$ be a compact Kähler manifold of complex dimension $n$ and $D$ a divisor of $\bar{X}$ with normal crossings. Let $E$ be a rank $r$ holomorphic vector bundle over $\bar{X}$ with a parabolic structure along $D$. If $E$ is parabolic stable, the following Chern number inequality holds.

$$
\begin{aligned}
\left(C_{1}^{2}-\right. & \left.2 C_{2}\right)+2 \sum_{i=1}^{m} \sum_{l=1}^{m_{i}} \alpha_{l}^{i} \operatorname{deg}\left(F_{l}^{i} / F_{l+1}^{i}\right)+\sum_{i=1}^{m} \sum_{l=1}^{m_{i}}\left(\alpha_{l}^{i}\right)^{2} \operatorname{rank}\left(F_{l}^{i} / F_{l+1}^{i}\right) D_{i}^{2} \\
\leq & \frac{1}{r}\left(C_{1}^{2}+2 \sum_{i=1}^{m} \sum_{l=1}^{m_{i}} \alpha_{l}^{i} \operatorname{rank}\left(F_{l}^{i} / F_{l+1}^{i}\right) \operatorname{deg}\left(\left.E\right|_{D_{i}}\right)\right. \\
& \left.+\sum_{i, j=1}^{m}\left(\sum_{l=1}^{m_{i}} \alpha_{l}^{i} \operatorname{rank}\left(F_{l}^{i} / F_{l+1}^{i}\right)\right)\left(\sum_{l=1}^{m_{j}} \alpha_{l}^{j} \operatorname{rank}\left(F_{l}^{j} / F_{l+1}^{j}\right)\right) D_{i} \cdot D_{j}\right)
\end{aligned}
$$

where $D_{i} \cdot D_{j}$ is the intersection number of $D_{i}$ and $D_{j}(i, j=1, \cdots, m)$, $D_{i}^{2}$ is the self-intersection number of $D_{i}, C_{2}=\int_{\bar{X}} C_{2}(E) \wedge \omega^{n-2}, C_{1}^{2}=$ $\int_{\bar{X}} C_{1}(E) \wedge C_{1}(E) \wedge \omega^{n-2}$. 
Proof. By Proposition 7.2, we have

$$
2 \int_{X} C_{2}\left(E, K_{0}\right) \wedge \omega_{\alpha}^{n-2} \geq \frac{r-1}{r} \int_{X} C_{1}\left(E, K_{0}\right) \wedge C_{1}\left(E, K_{0}\right) \wedge \omega_{\alpha}^{n-2}
$$

for any $2\left(1-\gamma_{0}\right) \leq \alpha<2$. that is,

$$
\begin{aligned}
-\left(\frac{\sqrt{-1}}{2 \pi}\right)^{2} \int_{X} \operatorname{tr}\left(F_{K_{0}} \wedge F_{K_{0}}\right) & \wedge \omega_{\alpha}^{n-2} \\
& \geq-\frac{1}{r}\left(\frac{\sqrt{-1}}{2 \pi}\right)^{2} \int_{X} t r F_{K_{0}} \wedge t r F_{K_{0}} \wedge \omega_{\alpha}^{n-2}
\end{aligned}
$$

so

$$
\begin{aligned}
\left(\frac{\sqrt{-1}}{2 \pi}\right)^{2} \int_{X} \operatorname{tr}\left(F_{K_{0}} \wedge F_{K_{0}}\right) & \wedge \omega_{\alpha}^{n-2} \\
& \leq \frac{1}{r}\left(\frac{\sqrt{-1}}{2 \pi}\right)^{2} \int_{X} \operatorname{tr} F_{K_{0}} \wedge \operatorname{tr} F_{K_{0}} \wedge \omega_{\alpha}^{n-2}
\end{aligned}
$$

Then the theorem follows from Lemma 7.3 and Lemma 7.4.

\section{Chern number inequality (II).}

In this section, we assume that $D$ is a divisor in $\bar{X}$ and that $D=\sum_{i=1}^{m} D_{i}$ where the irreducible components $D_{i}$ of $D$ are smooth, we do not assume that $D_{i}$ meat transversely. Let $E$ be a holomorphic vector bundle over $\bar{X}$, we shall define the notion of parabolic structure of $E$ along $D$ and the notion of parabolic stability for a parabolic bundle, we shall derive at last a Chern number inequality for a stable parabolic bundle.

Definition 8.1. A parabolic structure on $E$ with respect to $D$ consists of

a) flags of $\left.E\right|_{D_{i}}(i=1, \cdots, m)$,

$$
\left.E\right|_{D_{i}}=F_{1}^{i} \supset F_{2}^{i} \supset \cdots \supset F_{m_{i}}^{i} \supset\{0\}=F_{m_{i}+1}^{i}
$$

where $F_{l+1}^{i}$ is a proper subbundle of $F_{l}^{i}\left(l=1, \cdots, m_{i}-1\right)$.

b) weights $\alpha_{1}^{i}, \cdots, \alpha_{m_{i}}^{i}$ attached to $F_{1}^{i}, \cdots, F_{m_{i}}^{i}$ satisfying $0 \leq \alpha_{1}^{i}<\cdots<$ $\alpha_{m_{i}}^{i}<1$. 
A holomorphic vector bundle $E$ with a parabolic structure is called parabolic bundle.

We define the parabolic degree of a parabolic bundle $E$ by

$$
p a r \operatorname{deg} E=\operatorname{deg} E+\sum_{i=1}^{m} \sum_{l=1}^{m_{i}} \alpha_{l}^{i} \operatorname{rank}\left(F_{l}^{i} / F_{l+1}^{i}\right) \operatorname{deg}\left[D_{i}\right]
$$

Suppose that $V$ is a proper coherent subsheaf of $E$ with quotient torsion free. There is a natural flag of $\left.V\right|_{D_{i}}$ by coherent subsheaves

$$
\left.V\right|_{D_{i}}=F_{1}^{i} V \supset \cdots \supset F_{n_{i}}^{i} V \supset\{0\}=F_{n_{i}+1}^{i}
$$

induced by $F_{1}^{i} \cap V \supseteq \cdots \supseteq F_{m_{i}}^{i} \cap V \supset\{0\}$. We define the weights attached to the flag by $\alpha_{l}^{i}(V)=$ the largest $\alpha_{k}^{i}$ such that $F_{l}^{i} V \subseteq F_{k}^{i} \cap V, l=1, \cdots, n_{i}$. We define the parabolic degree of $V$ by

$$
p a r \operatorname{deg} V=\operatorname{deg} V+\sum_{i=1}^{m} \sum_{l=1}^{n_{i}} \alpha_{l}^{i}(V) \operatorname{rank}\left(F_{l}^{i} V / F_{l+1}^{i} V\right) \operatorname{deg}\left[D_{i}\right]
$$

Definition 8.2. We say that a parabolic bundle $E$ is parabolic stable if for every proper coherent subsheaf $V$ of $E$ with quotient torsion free we have

$$
\frac{\operatorname{par} \operatorname{deg} V}{\operatorname{rankV}}<\frac{\operatorname{par} \operatorname{deg} E}{\operatorname{rankE}}
$$

In this section we mainly prove the following Chern number inequality for a parabolic stable bundle.

Theorem 8.3. Let $\bar{X}$ be a compact Kähler manifold of complex dimension n. Let $D=\sum_{i=1}^{m} D_{i}$ be a divisor in $\bar{X}$ where the irreducible components $D_{i}$ of $D$ are smooth. Let $E$ be a rank $r$ holomorphic vector bundle over $\bar{X}$ with a parabolic structure along $D$. If $E$ is parabolic stable,

$$
\begin{aligned}
\left(C_{1}^{2}-\right. & \left.2 C_{2}\right)+2 \sum_{i=1}^{m} \sum_{l=1}^{m_{i}} \alpha_{l}^{i} \operatorname{deg}\left(F_{l}^{i} / F_{l+1}^{i}\right)+\sum_{i=1}^{m} \sum_{l=1}^{m_{i}}\left(\alpha_{l}^{i}\right)^{2} \operatorname{rank}\left(F_{l}^{i} / F_{l+1}^{i}\right) D_{i}^{2} \\
\leq & \frac{1}{r}\left(C_{1}^{2}+2 \sum_{i=1}^{m} \sum_{l=1}^{m_{i}} \alpha_{l}^{i} \operatorname{rank}\left(F_{l}^{i} / F_{l+1}^{i}\right) \operatorname{deg}\left(\left.E\right|_{D}\right)\right. \\
& \left.+\sum_{i, j=1}^{m}\left(\sum_{l=1}^{m_{i}} \alpha_{l}^{i} \operatorname{rank}\left(F_{l}^{i} / F_{l+1}^{i}\right)\right)\left(\sum_{l=1}^{m_{j}} \alpha_{l}^{j} \operatorname{rank}\left(F_{l}^{j} / F_{l+1}^{j}\right)\right) D_{i} \cdot D_{j}\right)
\end{aligned}
$$


where $D_{i} \cdot D_{j}$ is the intersection number of $D_{i}$ and $D_{j}(i, j=1, \cdots, m)$, $D_{i}^{2}$ is the self-intersection number of $D_{i}, C_{1}^{2}=\int_{\bar{X}} C_{1}(E) \wedge C_{1}(E) \wedge \omega^{n-2}$, $C_{2}=\int_{\bar{X}} C_{2}(E) \wedge \omega^{n-2}$.

Proof. We use the theorem of Hironaka ([H],p.145, Corollary 2) again. By successively blowing up complex submanifolds, we can find a complex manifold $\widetilde{X}$ such that the following holds. Let $q: \widetilde{X} \rightarrow X$ be the canonical map and let $\left\{P_{i}^{n_{i}}\right\}(i=1, \cdots, N)$ be the components of the exceptional divisor $S$. Let $D_{i}^{*}$ be the proper transform of $D_{i}(i=1, \cdots, m)$. We may assume that $D_{i}^{*}$ do not meet each other, and that $q^{-1}(D)=\sum_{i=1}^{m} D_{i}^{*}+S$ forms a divisor with normal crossings.

Note that $q^{*} \omega$ is a Kähler metric on $\widetilde{X} \backslash S$, but it is not a metric on $S$. Suppose that $\widetilde{\omega}$ is a Kähler metric on $\widetilde{X}$, then for any $\varepsilon>0, \omega_{\varepsilon}=q^{*} \omega+\varepsilon \widetilde{\omega}$ is a Kähler metric on $\widetilde{X}$.

The parabolic structure of $E$ along $D$ induces a parabolic structure of $q^{*} E$ along $D^{*}=\sum_{i=1}^{m} D_{i}^{*}$ which consists of

a') flags of $\left.q^{*} E\right|_{D_{i}^{*}}(i=1, \cdots, m)$

$$
\left.q^{*} E\right|_{D_{i}^{*}}=\left.\left.\left.q^{*} F_{1}^{i}\right|_{D_{i}^{*}} \supset q^{*} F_{2}^{i}\right|_{D_{i}^{*}} \supset \cdots q^{*} F_{m_{i}}^{i}\right|_{D_{i}^{*}} \supset\{0\}=\left.q^{*} F_{m_{i}+1}^{i}\right|_{D_{i}^{*}}
$$

b') weights $\alpha_{1}^{i}, \cdots, \alpha_{m_{i}}^{i}$ attached to the flags.

Set

$$
\begin{aligned}
\operatorname{par} \operatorname{deg} q^{*} E= & \operatorname{deg}_{q^{*} \omega} q^{*} E \\
& +\sum_{i=1}^{m} \sum_{l=1}^{m_{i}} \alpha_{l}^{i} \operatorname{rank}\left(\left.q^{*} F_{l}^{i}\right|_{D_{i}^{*}} /\left.q^{*} F_{l+1}^{i}\right|_{D_{i}^{*}}\right) \operatorname{deg}_{q^{*} \omega}\left[D_{i}^{*}\right]
\end{aligned}
$$

where

$$
\begin{aligned}
\operatorname{deg}_{q^{*} \omega} q^{*} E & =\int_{\widetilde{X}} C_{1}\left(q^{*} E\right) \wedge * q^{*} \omega \\
& =\int_{\bar{X}} C_{1}(E) \wedge \omega=\operatorname{deg} E \\
\operatorname{deg}_{q^{*} \omega}\left[D_{i}^{*}\right] & =\int_{\widetilde{X}} C_{1}\left(\left[D_{i}^{*}\right]\right) \wedge * q^{*} \omega=\operatorname{deg}\left[D_{i}\right]
\end{aligned}
$$

so

par $\operatorname{deg} q^{*} E=\operatorname{par} \operatorname{deg} E$. 
Put

$$
\begin{aligned}
\operatorname{par} \operatorname{deg}_{\varepsilon} q^{*} E= & \operatorname{deg}_{\omega_{\varepsilon}} q^{*} E \\
& +\sum_{i=1}^{m} \sum_{l=1}^{m_{i}} \alpha_{l}^{i} \operatorname{rank}\left(\left.q^{*} F_{l}^{i}\right|_{D_{i}^{*}} / q^{*} F_{l+1}^{i} \mid D_{i}^{*}\right) \operatorname{deg}_{\omega_{\varepsilon}}\left[D_{i}^{*}\right]
\end{aligned}
$$

where

$$
\begin{aligned}
& \operatorname{deg}_{\omega_{\varepsilon}} q^{*} E=\int_{\widetilde{X}} C_{1}\left(q^{*} E\right) \wedge *\left(q^{*} \omega+\varepsilon \widetilde{\omega}\right)=\operatorname{deg} E+\delta_{0}(\varepsilon) \\
& \operatorname{deg}_{\omega_{\varepsilon}}\left[D_{i}^{*}\right]=\int_{\widetilde{X}} C_{1}\left(\left[D_{i}^{*}\right]\right) \wedge *\left(q^{*} \omega+\varepsilon \widetilde{\omega}\right)=\operatorname{deg}\left[D_{i}\right]+\delta_{i}(\varepsilon)
\end{aligned}
$$

and $\delta_{i}(\varepsilon) \rightarrow 0$ as $\varepsilon \rightarrow 0(i=0, \cdots, m)$.

So $\operatorname{par} \operatorname{deg}_{\varepsilon} q^{*} E=$ par deg $E+\delta(\varepsilon)$ where $\delta(\varepsilon) \rightarrow 0$ as $\varepsilon \rightarrow 0$.

Let $V^{*}$ be a proper coherent subsheaf of $q^{*} E$ with quotient torsion free. $\left.\left(q^{-1}\right)^{*} V^{*}\right|_{q^{-1}(\tilde{X} \backslash S)}$ can be extended to $\bar{X}$ as a coherent subsheaf of $E$, we denote it by $V$. Similarly, we can define $\operatorname{par} \operatorname{deg} V^{*}$ and $\operatorname{par} \operatorname{deg}_{\varepsilon} V^{*}$ by

$$
\begin{aligned}
\operatorname{par} \operatorname{deg} V^{*}= & \operatorname{deg}_{q^{*} \omega} V^{*} \\
& +\sum_{i=1}^{m} \sum_{l=1}^{n_{i}} \alpha_{l}^{i}\left(V^{*}\right) \operatorname{rank}\left(\left.q^{*} F_{l}^{i}\right|_{D_{i}^{*}}\left(V^{*}\right) /\left.q^{*} F_{l+1}^{i}\right|_{D_{i}^{*}}\left(V^{*}\right)\right) \operatorname{deg}_{q^{*} \omega}\left[D_{i}^{*}\right]
\end{aligned}
$$

and

$$
\begin{aligned}
\operatorname{par} \operatorname{deg}_{\varepsilon} V^{*}= & \operatorname{deg}_{\omega_{\varepsilon}} V^{*} \\
& +\sum_{i=1}^{m} \sum_{l=1}^{n_{i}} \alpha_{l}^{i}\left(V^{*}\right) \operatorname{rank}\left(\left.q^{*} F_{l}^{i}\right|_{D_{i}^{*}}\left(V^{*}\right) /\left.q^{*} F_{l+1}^{i}\right|_{D_{i}^{*}}\left(V^{*}\right)\right) \operatorname{deg}_{\omega_{\varepsilon}}\left[D_{i}^{*}\right]
\end{aligned}
$$

It is clear that par $\operatorname{deg} V^{*}=p a r \operatorname{deg} V$ and $\operatorname{par}_{\operatorname{deg}} V^{*}=p a r \operatorname{deg} V+\eta(\varepsilon)$, where $\eta(\varepsilon) \rightarrow 0$ as $\varepsilon \rightarrow 0$.

The following lemma is obvious.

Lemma 8.4. $E$ is parabolic stable if and only if $q^{*} E$ is parabolic stable with respect to $q^{*} \omega$.

Furthermore, we have

Lemma 8.5. Suppose that $E$ is parabolic stable. Then $q^{*} E$ is parabolic stable with respect to $\omega_{\varepsilon}$ for sufficiently small $\varepsilon$. 
Proof. If $q^{*} E$ were not parabolic stable with respect to $\omega_{\varepsilon}$, there would exist a proper coherent subsheaf $V^{*}$ of $q^{*} E$ with quotient torsion free such that

$$
\frac{\operatorname{par} \operatorname{deg}_{\varepsilon} V^{*}}{\operatorname{rank} V^{*}} \geq \frac{\operatorname{par} \operatorname{deg}_{\varepsilon} q^{*} E}{\operatorname{rankq}^{*} E}
$$

On the other hand, since $q^{*} E$ is parabolic stable with respect to $q^{*} \omega$ (Lemma 8.4), we have

$$
\frac{\operatorname{par} \operatorname{deg} V^{*}}{\operatorname{rank} V^{*}}<\frac{\operatorname{par} \operatorname{deg} q^{*} E}{\operatorname{rank} q^{*} E}
$$

We consider $\operatorname{deg}_{q^{*} \varepsilon}\left(\left(V^{*}\right)^{*} \otimes q^{*} E\right)$.

By (9), we have

$$
\begin{aligned}
\operatorname{deg}_{q^{*} \omega} & \left(\left(V^{*}\right)^{*} \otimes q^{*} E\right) \\
= & \operatorname{rank} V^{*} \operatorname{deg}_{q^{*} \omega} q^{*} E-\operatorname{rank} q^{*} E \operatorname{deg}_{q^{*} \omega} V^{*} \\
= & \operatorname{rank} V^{*} \operatorname{rank} q^{*} E\left(\frac{\operatorname{deg}_{q^{*} \omega} q^{*} E}{\operatorname{rank} q^{*} E}-\frac{\operatorname{deg}_{q^{*} \omega} V^{*}}{\operatorname{rank} V^{*}}\right) \\
\leq & \operatorname{rank} V^{*} \operatorname{rank} q^{*} E\left(\frac { 1 } { \operatorname { r a n k } V ^ { * } } \sum _ { i = 1 } ^ { m } \sum _ { l = 1 } ^ { n _ { i } } \left(\alpha_{l}^{i}\left(V^{*}\right)\right.\right. \\
& \left.\cdot \operatorname{rank}\left(\left.q^{*} F_{l}^{i}\right|_{D_{i}^{*}}\left(V^{*}\right) / q^{*} F_{l+1}^{i} \mid D_{i}^{*}\left(V^{*}\right)\right)\right) \operatorname{deg}_{q^{*} \omega}\left[D_{i}^{*}\right] \\
& \left.-\frac{1}{\operatorname{rank} q^{*} E} \sum_{i=1}^{m} \sum_{l=1}^{m_{i}} \alpha_{l}^{i} \operatorname{rank}\left(\left.q^{*} F_{l}^{i}\right|_{D_{i}^{*}} /\left.q^{*} F_{l+1}^{i}\right|_{D_{i}^{*}}\right) \operatorname{deg}_{q^{*} \omega}\left[D_{i}^{*}\right]\right) \\
& +C(\varepsilon)
\end{aligned}
$$

where $C(\varepsilon) \rightarrow 0$ as $\varepsilon \rightarrow 0$.

By (10), we have

$$
\begin{aligned}
\operatorname{deg}_{q^{*} \omega} & \left(\left(V^{*}\right)^{*} \otimes q^{*} E\right) \\
> & \operatorname{rankV}^{*} \operatorname{rank}^{*} E\left(\frac { 1 } { \operatorname { r a n k V } } \sum _ { i = 1 } ^ { m } \sum _ { l = 1 } ^ { n _ { i } } \left(\alpha_{l}^{i}\left(V^{*}\right)\right.\right. \\
& \left.\cdot \operatorname{rank}\left(\left.q^{*} F_{l}^{i}\right|_{D_{i}^{*}}\left(V^{*}\right) /\left.q^{*} F_{l+1}^{i}\right|_{D_{i}^{*}}\left(V^{*}\right)\right)\right) \operatorname{deg}_{q^{*} \omega}\left[D_{i}^{*}\right] \\
& \left.\quad-\frac{1}{\operatorname{rank} q^{*} E} \sum_{i=1}^{m} \sum_{l=1}^{m_{i}} \alpha_{l}^{i} \operatorname{rank}\left(\left.q^{*} F_{l}^{i}\right|_{D_{i}^{*}} /\left.q^{*} F_{l+1}^{i}\right|_{D_{i}^{*}}\right) \operatorname{deg}_{q^{*} \omega}\left[D_{i}^{*}\right]\right)
\end{aligned}
$$

(11) contradicts (12) when $\varepsilon$ is sufficiently small, because $\operatorname{deg}_{q^{*} \omega}\left(\left(V^{*}\right)^{*} \otimes\right.$ $\left.q^{*} E\right)=\operatorname{deg}\left(V^{*} \otimes E\right)$ is an integer. This completes the proof of the lemma. 
Now we can finish the proof of Theorem 8.3.

Since $\left(\widetilde{X}, \omega_{\varepsilon}\right)$ is a compact Kähler manifold, $D^{*}=\sum_{i=1}^{m} D_{i}^{*}$ is a divisor in $\widetilde{X}$, and $D_{i}^{*}(i=1, \cdots, m)$ do not meet each other. Since $E$ is parabolic stable, $q^{*} E$ is parabolic stable with respect to $\omega_{\varepsilon}$ for sufficiently small $\varepsilon$. By Theorem 7.5 we have

$$
\begin{aligned}
\left(C_{1}^{2, \varepsilon}-2 C_{2}^{\varepsilon}\right)+2 \sum_{i=1}^{m} \sum_{l=1}^{m_{i}} \alpha_{l}^{i} \operatorname{deg}_{\omega_{\varepsilon}}\left(\left.q^{*} F_{l}^{i}\right|_{D_{i}^{*}} /\left.q^{*} F_{l+1}^{i}\right|_{i} ^{*}\right) \\
+\sum_{i=1}^{m} \sum_{l=1}^{m_{i}}\left(\alpha_{l}^{i}\right)^{2} \operatorname{rank}\left(\left.q^{*} F_{l}^{i}\right|_{D_{i}^{*}} /\left.q^{*} F_{l+1}^{i}\right|_{D_{i}^{*}}\right) \\
\cdot \int_{\tilde{X}} C_{1}\left(\left[D_{i}^{*}\right]\right) \wedge C_{1}\left(\left[D_{i}^{*}\right]\right) \wedge \omega_{\varepsilon}^{n-2} \\
\leq \frac{1}{r}\left(C_{1}^{2, \varepsilon}+2 \sum_{i=1}^{m} \sum_{l=1}^{m_{i}} \alpha_{l}^{i} \operatorname{rank}\left(\left.q^{*} F_{l}^{i}\right|_{D_{i}^{*}} /\left.q^{*} F_{l+1}^{i}\right|_{D_{i}^{*}}\right) \operatorname{deg}_{\omega_{\varepsilon}}\left(\left.E\right|_{D_{i}^{*}}\right)\right. \\
+\sum_{i, j=1}^{m}\left(\sum_{l=1}^{m_{i}} \alpha_{l}^{i} \operatorname{rank}\left(\left.q^{*} F_{l}^{i}\right|_{D_{i}^{*}} / q^{*} F_{l+1}^{i} \mid D_{i}^{*}\right)\right) \\
\cdot\left(\sum_{l=1}^{m_{j}} \alpha_{l}^{j} \operatorname{rank}\left(\left.q^{*} F_{l}^{j}\right|_{D_{j}^{*}} /\left.q^{*} F_{l+1}^{j}\right|_{D_{j}^{*}}\right)\right) \\
\left.\cdot \int_{\tilde{X}} C_{1}\left(\left[D_{i}^{*}\right]\right) \wedge C_{1}\left(\left[D_{j}^{*}\right]\right) \wedge \omega_{\varepsilon}^{n-2}\right)
\end{aligned}
$$

where

$$
\begin{aligned}
C_{1}^{2, \varepsilon} & =\int_{\widetilde{X}} C_{1}\left(q^{*} E\right) \wedge C_{1}\left(q^{*} E\right) \wedge \omega_{\varepsilon}^{n-2} \\
C_{2}^{\varepsilon} & =\int_{\tilde{X}} C_{2}\left(q^{*} E\right) \wedge \omega_{\varepsilon}^{n-2}
\end{aligned}
$$

Letting $\varepsilon \rightarrow 0$, we get the desired inequality. This completes the proof of the theorem.

\section{References.}

[B] O. Biquard, Fibrés parabolique stables et connexions singulières plates, Bull. Soc. Math. France, 119 (1991), 231-157.

[D1] S.K. Donaldson, A new proof of a theorem of Narasimhan and Seshadri, J. Diff. Geom. 18 (1983), 269-277. 
[D2] S.K. Donaldson, Anti-self-dual Yang-Mills connections over complex algebraic surfaces and stable bundles, Proc. London Math. Soc. 50 (1985), 1-26.

[D3] S.K. Donaldson, Infinite determinants, stable bundles and curvature, Duke Math. J. 54 (1987), 231-247.

[D4] S.K. Donaldson, Boundary value problems for Yang-Mills fields, J. Geom. and Phy. 8 (1992), 89-122.

[G-T] D. Gilbarg and N.S. Trudinger, Elliptic Partial Differential Equations of Second Order, Springer-Verlag, 1977.

[H] H. Hironaka, Resolution of singularities of an algebraic variety over a field of characteristic zero, Ann. of Math. 79 (1964), 109-326.

[K] H. Konno, Construction of the moduli space of stable parabolic Higgs bundles on a Riemann surface, J. Math. Soc. Japan, 45 (1993), 253-276.

[L-N] J. Li and M.S. Narasimhan, Hermitian-Einstein metrics on parabolic stable bundles, Preprint, ICTP, 1995.

[M-S] V. Mehta and C.S. Seshadri, Moduli of vector bundles on curves with parabolic structures, Math. Ann. 248 (1980), 205-239.

[Mo] J. Moser, On Harnack's theorem for elliptic differential equations, Comm. Pure Appl. Math. 14 (1961), 577-591.

[N-S] M.S. Narasimhan and C.S. Seshadri, Stable and unitary vector bundles on a compact Riemann surface, Ann. of Math. 82 (1965), 540-567.

[Na-St] E.B. Nasatyr and B. Steer, The Narasimhan-Seshadri theorem for parabolic bundles with irrational weights, preprint.

[P] J.A. Poritz, Parabolic vector bundles and Hermitian-Yang-Mills connections over a Riemann surface, Inter. J. Math. 4 (1993), 467-501.

[S1] C.T. Simpson, Constructing variation of Hodge structure using Yang-Mills theory and applications to uniformization, J. Amer. Math. Soc. 1 (1988), 867-918.

[S2] C.T. Simpson, Harmonic bundles on noncompact curves, J. Amer. Math. Soc. 3 (1990), 713-770.

[Siu] Y.T. Siu, Techniques of Extension of Analytic Objects, Lect. Notes in Pure and Appl. Math., Vol. 8, Marcel Dekker, Inc., New York, 1974.

[SABK] C. Soulé, D. Abramovich, J.-F. Burnol and J. Kramer, Lectures on Arakelov Geometry, Cambridge studies in advanced math. 33, Cambridge University Press, 1992. 
[St] E.W. Stredulinsky, Weighted Inequalities and Degenerate Elliptic Partial Differential Equations, Lecture Notes in Math. 1074, Springer-Verlag, 1984.

[U-Y] K.K. Uhlenbeck and S.T. Yau, On the existence of Hermitian Yang-Mills connections in stable bundles, Comm. Pure Appl. Math. 39-S (1986), 257293.

INSTITUTE OF MATHEMATICS

ACADEMIa Sinica

BEIJING 100080

P.R.OF CHINA

RECEIVED SePtember 24, 1997. 